\title{
PARABOLIC FLOWS ON ALMOST HERMITIAN MANIFOLDS
}

\author{
Masaya KAWAMURA \\ (Received 6 September 2017 and revised 10 April 2018)
}

\begin{abstract}
We define two parabolic flows on compact almost Hermitian manifolds, which coincide with the pluriclosed flow and the Hermitian curvature flow, respectively, on compact Hermitian manifolds with pluriclosed metric. We study the relation between these two parabolic evolution equations.
\end{abstract}

\section{Introduction}

In [4] and [6], Streets and Tian introduced a parabolic evolution equation of pluriclosed metrics on a compact Hermitian manifold, which is called the pluriclosed flow. At first, they asked whether or not it is possible to prove classification results in higher dimensions for complex non-Kähler manifolds using geometric evolution equations as in the case that the Ricci flow was used for proving uniformization of Riemann surfaces. They tried to have a parabolic flow such that it preserves Hermitianness and as much additional structure as possible and also is as close to the Kähler-Ricci flow as possible. Since a pluriclosed form $\omega$ is locally given by $\omega=\partial \eta+\bar{\partial} \bar{\eta}$ for some $\eta \in \Lambda^{0,1}$ (cf. [3, Lemma 3.9]), they concluded that it is natural to define a flow of pluriclosed metrics using a second-order closed $(1,1)$-form (the Chern curvature form) and a first-order $(0,1)$-form. From this point of view, they defined the pluriclosed flow starting at a pluriclosed metric. They also introduced the Hermitian curvature flow with a quadratic $Q^{1}$ in the torsion of the Chern connection in [4]: let $(M, J)$ be a compact complex manifold with pluriclosed metric $g_{0}$. They have proven that the following evolution equation has a unique solution $g(t)$ on $M$ (cf. [4, Theorem 1.2]):

$$
\left\{\begin{array}{l}
\frac{\partial}{\partial t} g(t)=-S(g(t))+Q^{1}(g(t)), \\
g(0)=g_{0} .
\end{array}\right.
$$

Streets and Tian showed that the solution of the pluriclosed flow coincides with the solution of $(\mathrm{HCF})_{Q^{1}}$ as follows (cf. [4, Proposition 3.3]).

Proposition 1.1. (Streets-Tian identifiability theorem) Let $(M, J)$ be a compact complex manifold with pluriclosed metric $\tilde{g}$. The solution to $(H C F)_{Q^{1}}$ with pluriclosed initial condition $\tilde{g}$ is equivalent to a solution to the pluriclosed flow with the initial condition $\tilde{\omega}$ on $M$, where $\tilde{\omega}$ is the associated real $(1,1)$-form with respect to $\tilde{g}$.

2010 Mathematics Subject Classification: Primary 53C44; Secondary 53C55; 32W20.

Keywords: almost Hermitian metrics; parabolic evolution equation; Chern connection.

(C) 2019 Faculty of Mathematics, Kyushu University 
This result indicates that the flow $(\mathrm{HCF})_{Q^{1}}$ preserves the pluriclosed condition. It is because the pluriclosed flow preserves the pluriclosed condition and the solution to the pluriclosed flow solves $(\mathrm{HCF})_{Q^{1}}$ (Streets-Tian identifiability theorem), and moreover, since the solutions are unique, a solution to $(\mathrm{HCF})_{Q^{1}}$ with the pluriclosed initial condition coincides with a solution to the pluriclosed flow with the same initial metric, and hence the flow $(\mathrm{HCF})_{Q^{1}}$ must preserve the pluriclosed condition. Our approach is to try to generalize their flow to almost Hermitian cases and to expect to obtain similar results as in the complex cases. In this paper, we would like to define two parabolic flows and show that the identifiability theorem holds for these flows on compact almost Hermitian manifolds as well.

Let $(M, J)$ be a compact almost complex manifold and let $g$ be an almost Hermitian metric on $M$. Let $\left\{Z_{r}\right\}$ be an arbitrary local $(1,0)$-frame around a fixed point $p \in M$ and let $\left\{\zeta^{r}\right\}$ be the associated coframe. Then the associated unique real $(1,1)$-form $\omega$ with respect to $g$ takes the local expression $\omega=\sqrt{-1} g_{r \bar{k}} \zeta^{r} \wedge \zeta^{\bar{k}}$. We will also refer to $\omega$ as an almost Hermitian metric. We would like to define a parabolic flow of almost Hermitian metrics with an almost Hermitian initial metric $\omega_{0}$ on $(M, J)$. We will call it the almost Hermitian flow (AHF):

$$
\left\{\begin{array}{l}
\frac{\partial}{\partial t} \omega(t)=\partial \partial_{g(t)}^{*} \omega(t)+\bar{\partial} \bar{\partial}_{g(t)}^{*} \omega(t)-P(\omega(t))=:-\Phi(\omega(t)), \\
\omega(0)=\omega_{0}
\end{array}\right.
$$

where $\partial_{g(t)}^{*}$ and $\bar{\partial}_{g(t)}^{*}$ are the $L^{2}$-adjoint operators with respect to metrics $g(t)$, and $P(\omega)$ is one of the Ricci-type curvatures of the Chern curvature. One has with an arbitrary $(1,0)$-frame $\left\{Z_{r}\right\}$ with respect to $g, P_{i \bar{j}}=g^{k \bar{l}} \Omega_{i \bar{j} k \bar{l}}$, where $\Omega$ is the curvature of the Chern connection $\nabla$ on $(M, g, J)$.

Our first goal is to prove that the operator $\omega \mapsto \Phi(\omega)$ is a strictly elliptic operator for an almost Hermitian metric $\omega$, which means that the equation (AHF) with an almost Hermitian initial metric is a strictly parabolic equation. Hence the short-time existence and the uniqueness of the solution (AHF) follows from the standard parabolic theory since the manifold is supposed to be compact. This flow (AHF) coincides with the pluriclosed flow if $J$ is integrable and also the initial metric is pluriclosed.

THEOREM 1.1. Given a compact almost Hermitian manifold $\left(M, \omega_{0}, J\right)$, there exists a unique solution to $(A H F)$ with initial condition $\omega_{0}$ on $[0, \varepsilon)$ for some $\varepsilon>0$.

The following result indicates that the parabolic flow (AHCF) introduced in the statement of Theorem 1.2 could play the same role as the flow $(\mathrm{HCF})_{Q^{1}}$ on complex manifolds. We may expect to have some other similar results as in $[4,5]$ and $[\mathbf{6}]$ for $(\mathrm{AHCF})$. Our second goal is to show the following generalized Streets-Tian identifiability theorem. We denote by $S$ one of the Ricci-type curvatures of the Chern curvature, which is locally given by $S_{i \bar{j}}=g^{k \bar{l}} \Omega_{k \bar{l}} \bar{j}$.

THEOREM 1.2. Let $\left(M, g_{0}, J\right)$ be a compact almost Hermitian manifold with fundamental form $\omega_{0}$. Then the metric $g_{t}$ of the solution $\omega_{t}$ to $(A H F)$ starting at $\omega_{0}$ evolves as

$$
\left\{\begin{array}{l}
\frac{\partial}{\partial t} g(t)=-S(g(t))-Q^{7}(g(t))-Q^{8}(g(t))+B T^{\prime}(g(t))+\bar{Z}\left(T^{\prime}\right)(g(t)), \\
g(0)=g_{0},
\end{array}\right.
$$


where $w_{i}:=T_{i r \bar{r}}$,

$$
B T_{i \bar{j}}^{\prime}:=B_{\bar{r} p}^{j} T_{i r \bar{p}}+B_{\bar{p} i}^{r} T_{p r} \bar{j}+B_{\bar{r} r}^{p} T_{p i \bar{j}}+B_{\bar{j} i}^{r} w_{r}
$$

and

$$
\bar{Z}\left(T^{\prime}\right)_{i \bar{j}}:=-Z_{\bar{r}}\left(T_{r i}^{s}\right) g_{s \bar{j}}-Z_{\bar{j}}\left(w_{i}\right)-g^{p \bar{q}} T_{p i}^{r} Z_{\bar{j}}\left(g_{r \bar{q}}\right) .
$$

Here $T$ is the torsion of the Chern connection and the components are computed with respect to a unitary frame. Then a solution to (AHCF) with initial condition $\omega_{0}$ is equivalent to a solution to $(A H F)$ starting at the same initial condition $\omega_{0}$.

In the sequel we refer to (AHCF) as almost Hermitian curvature flow. Note that this result in Theorem 1.2 implies that there exists a unique short-time solution to (AHCF) with initial almost Hermitian metric on a compact almost Hermitian manifold by applying Theorem 1.1. The parabolic flow $(\mathrm{AHCF})$ coincides with the flow $(\mathrm{HCF})_{Q^{1}}$ starting at a pluriclosed metric $\omega_{0}$ if $J$ is integrable.

PROPOSITION 1.2. The parabolic flow $(A H C F)$ coincides with the flow $(H C F)_{Q^{1}}$ starting at a pluriclosed metric if $J$ is integrable.

Proof. Under our assumption that $J$ is integrable, we have $Q^{7}=Q^{8}=B T^{\prime}=0$. Also, since we have $\partial_{\bar{r}} T_{r i \bar{j}}=\partial_{\bar{j}} T_{r i \bar{r}}$ for a pluriclosed metric on a Hermitian manifold (one can check that (HCF) preserves the pluriclosedness (cf. [4, Theorem 3.4])) and then we may choose a local $(1,0)$-frame $Z_{r}=\partial / \partial z_{r}$ for some complex local coordinate $\left\{z_{1}, \ldots, z_{n}\right\}$, we obtain

$$
\begin{aligned}
\bar{Z}\left(T^{\prime}\right)_{i \bar{j}} & =-\partial_{\bar{r}}\left(T_{r i}^{s}\right) g_{s \bar{j}}-\partial_{\bar{j}}\left(w_{i}\right)-g^{p \bar{q}} T_{p i}^{r} \partial_{\bar{j}} g_{r \bar{q}} \\
& =-\partial_{\bar{r}} T_{r i \bar{j}}+T_{r i}^{s} \partial_{\bar{r}} g_{s \bar{j}}-\partial_{\bar{j}} T_{i r \bar{r}}-g^{p \bar{q}} T_{p i}^{r} \Gamma_{\bar{j} \bar{q}}^{\bar{k}} g_{r \bar{k}} \\
& =-\partial_{\bar{j}} T_{r i \bar{r}}-\partial_{\bar{j}} T_{i r \bar{r}}+T_{r i}^{s} \Gamma_{\bar{r} \bar{j}}^{\bar{k}} g_{s \bar{k}}-T_{r i}^{s} \Gamma_{\bar{j} \bar{r} \bar{k}}^{\bar{k}} g_{s \bar{k}} \\
& =T_{i r \bar{s}} T_{\bar{j} \bar{r} s} \\
& =Q_{i \bar{j}}^{1},
\end{aligned}
$$

where we are writing $\partial_{r}, \partial_{\bar{j}}$ for $\partial / \partial z_{r}, \partial / \partial z_{\bar{j}}$, respectively. Combining these yields the result.

The result in Proposition 1.2 tells us that our flow (AHCF) can be considered as a generalized flow of the pluriclosed flow and $(\mathrm{HCF})_{Q^{1}}$. Here, concerning the difference between the flow (AHCF) and Vezzoni's flow in [8], Vezzoni studied the parabolic flow on a compact almost Hermitian manifold $\left(M^{2 n}, g_{0}, J\right)$ such that

$$
\frac{\partial}{\partial t} g(t)=-S-Q^{7}-Q^{8}+Q=:-K
$$

with $g(0)=g_{0}$, where $Q^{1}, Q^{2}, Q^{3}, Q^{4}, Q^{7}, Q^{8}$ are quadratics in the torsion of the Chern connection (cf. [8, p. 712])

$$
Q_{i \bar{j}}^{1}:=T_{i k \bar{r}} T_{\bar{j} \bar{k} r}, \quad Q_{i \bar{j}}^{2}:=T_{\bar{k} \bar{r} i} T_{k r \bar{j}}, \quad Q_{i \bar{j}}^{3}:=T_{i k \bar{k}} T_{\bar{j} \bar{r} r},
$$

and

$$
Q_{i \bar{j}}^{4}:=\frac{1}{2}\left(T_{r k \bar{k}} T_{\bar{r} \bar{j} i}+T_{\bar{r} \bar{k} k} T_{r i \bar{j}}\right), \quad Q_{i \bar{j}}^{7}:=T_{i r k} T_{\bar{r} \bar{k} \bar{j}}, \quad Q_{i \bar{j}}^{8}:=T_{i r k} T_{\bar{j} \bar{k} \bar{r}}
$$


and $Q:=\frac{1}{2} Q^{1}-\frac{1}{4} Q^{2}-\frac{1}{2} Q^{3}+Q^{4}$. These components are defined using an arbitrary unitary frame. Vezzoni's flow was defined for generalizing some studies on $(\mathrm{HCF})_{Q}$ in [5] and Hermitian Hilbert functional:

$$
\mathbb{F}(g)=\operatorname{Vol}(M)^{(1-n) / n} \int_{M} k d V
$$

where $k=\operatorname{tr}_{g} K=s-\frac{1}{4}\left|T^{\prime}\right|^{2}-\frac{1}{2}|w|^{2}$, where $s$ is the scalar curvature of the Chern connection. Note that we have $\operatorname{tr}_{g}\left(Q^{7}+Q^{8}\right)=0$. Vezzoni showed that a metric $g$ is a critical point of $\mathbb{F}$ if and only if $k$ is constant and $K=(k / n) g$. Our approach is different from this and it is to try to generalize the Streets-Tian identifiability theorem in [4]. For this purpose, we first showed that $\operatorname{div}^{\nabla} T^{\prime}=-\bar{\nabla} w-B T^{\prime}-\bar{Z}\left(T^{\prime}\right), \partial \partial_{g}^{*} \omega=-\nabla \bar{w}$ for any almost Hermitian metric $\omega$, and using these formulae, we generalized the identifiability theorem. This generalized result indicates that (AHCF) could possibly play the same role as the flow $(\mathrm{HCF})_{Q^{1}}$ and the pluriclosed flow. We may expect to have some other similar results as in [4] for (AHCF).

This paper is organized as follows. In Section 2, we recall some basic definitions and computations. In Section 3, we show the short-time existence and uniqueness of (AHF). Finally, in Section 4, we prove the generalized Streets-Tian identifiability theorem. Note that we assume the Einstein convention omitting the symbol of sum over repeated indices in all this paper.

\section{Preliminaries}

\subsection{The Nijenhuis tensor of the almost complex structure}

Let $M$ be a $2 n$-dimensional smooth differentiable manifold. An almost complex structure on $M$ is an endomorphism $J$ of $T M, J \in \Gamma(\operatorname{End}(T M))$, satisfying $J^{2}=-\operatorname{Id}_{T M}$. The pair $(M, J)$ is called an almost complex manifold. Let $(M, J)$ be an almost complex manifold. We define a bilinear map on $C^{\infty}(M)$ for $X, Y \in \Gamma(T M)$ by

$$
4 N(X, Y):=[J X, J Y]-J[J X, Y]-J[X, J Y]-[X, Y],
$$

which is the Nijenhuis tensor of $J$. The Nijenhuis tensor $N$ satisfies $N(X, Y)=-N(Y, X)$, $N(J X, Y)=-J N(X, Y), N(X, J Y)=-J N(X, Y), N(J X, J Y)=-N(X, Y)$. For any $(1,0)$-vector fields $W$ and $V, N(V, W)=-[V, W]^{(0,1)}, \quad N(V, \bar{W})=N(\bar{V}, W)=0$ and $N(\bar{V}, \bar{W})=-[\bar{V}, \bar{W}]^{(1,0)}$ since we have $4 N(V, W)=-2([V, W]+\sqrt{-1} J[V, W])$, $4 N(\bar{V}, \bar{W})=-2([\bar{V}, \bar{W}]-\sqrt{-1} J[\bar{V}, \bar{W}])$. An almost complex structure $J$ is called integrable if $N=0$ on $M$. Giving a complex structure to a differentiable manifold $M$ is equivalent to giving an integrable almost complex structure to $M$. Let $(M, J)$ be an almost complex manifold. A Riemannian metric $g$ on $M$ is called $J$-invariant if $J$ is compatible with $g$, i.e., for any $X, Y \in \Gamma(T M), g(X, Y)=g(J X, J Y)$. In this case, the pair $(g, J)$ is called an almost Hermitian structure. The fundamental 2-form $\omega$ associated to a $J$-invariant Riemannian metric $g$, i.e., an almost Hermitian metric, is determined by, for $X, Y \in \Gamma(T M)$, $\omega(X, Y)=g(J X, Y)$. Indeed we have, for any $X, Y \in \Gamma(T M)$,

$$
\omega(Y, X)=g(J Y, X)=g\left(J^{2} Y, J X\right)=-g(J X, Y)=-\omega(X, Y)
$$


and $\omega \in \Gamma\left(\bigwedge^{2} T^{*} M\right)$. We will also refer to the associated real fundamental $(1,1)$-form $\omega$ as an almost Hermitian metric. The form $\omega$ is related to the volume form $d V_{g}$ by $n ! d V_{g}=\omega^{n}$. Let $\left\{Z_{r}\right\}$ be a local $(1,0)$-frame on $(M, J)$ with an almost Hermitian metric $g$ and let $\{\zeta\}$ be a local associated coframe with respect to $\left\{Z_{r}\right\}$, i.e.,

$$
\zeta^{i}\left(Z_{j}\right)=\delta_{j}^{i}, \quad i, j=1, \ldots, n .
$$

Since $g$ is almost Hermitian, its components satisfy $g_{i j}=g_{\bar{i} \bar{j}}=0$ and $g_{i \bar{j}}=g_{\bar{j} i}=\bar{g}_{\bar{i} j}$.

Using the local frame $\left\{Z_{r}\right\}$ and coframe $\{\zeta\}$, we have

$$
N\left(Z_{\bar{i}}, Z_{\bar{j}}\right)=-\left[Z_{\bar{i}}, Z_{\bar{j}}\right]^{(1,0)}=: N_{\bar{i} \bar{j}}^{k} Z_{k}, \quad N\left(Z_{i}, Z_{j}\right)=-\left[Z_{i}, Z_{j}\right]^{(0,1)}=\overline{N_{\bar{i} j}^{k}} Z_{\bar{k}},
$$

and

$$
N=\frac{1}{2} \overline{N_{\bar{i}}^{k}} Z_{\bar{k}} \otimes\left(\zeta^{i} \wedge \zeta^{j}\right)+\frac{1}{2} N_{\bar{i} \bar{j}}^{k} Z_{k} \otimes\left(\zeta^{\bar{i}} \wedge \zeta^{\bar{j}}\right) .
$$

We write $T^{\mathbb{R}} M$ for the real tangent space of $M$. Then its complexified tangent space is given by

$$
T^{\mathbb{C}} M=T^{\mathbb{R}} M \otimes_{\mathbb{R}} \mathbb{C} .
$$

By extending $J \mathbb{C}$-linearly and $g, \omega \mathbb{C}$-bilinearly to $T^{\mathbb{C}} M$, they are also defined on $T^{\mathbb{C}} M$ and we observe that the complexified tangent space $T^{\mathbb{C}} M$ can be decomposed as

$$
T^{\mathbb{C}} M=T^{1,0} M \oplus T^{0,1} M,
$$

where $T^{1,0} M$ and $T^{0,1} M$ are the eigenspaces of $J$ corresponding to eigenvalues $\sqrt{-1}$ and $-\sqrt{-1}$, respectively:

$$
T^{1,0} M=\{X-\sqrt{-1} J X \mid X \in T M\}, \quad T^{0,1} M=\{X+\sqrt{-1} J X \mid X \in T M\} .
$$

Let $\Lambda^{r}(M)=\bigoplus_{p+q=r} \Lambda^{p, q}(M)$ for $0 \leq r \leq 2 n$ denote the decomposition of complex differential $r$-forms into $(p, q)$-forms, where $\Lambda^{p, q}(M)=\Lambda^{p}\left(\Lambda^{1,0} M\right) \otimes \Lambda^{q}\left(\Lambda^{0,1} M\right)$,

$$
\Lambda^{1,0} M=\left\{\eta+\sqrt{-1} J \eta \mid \eta \in \Lambda^{1} M\right\}, \quad \Lambda^{0,1} M=\left\{\eta-\sqrt{-1} J \eta \mid \eta \in \Lambda^{1} M\right\}
$$

and $\Lambda^{1} M$ denotes the dual of $T M$.

We define the adjoint operator $\partial_{g}^{*}$ of $\partial$ with respect to an almost Hermitian metric $g$ by

$$
\left(\partial_{g}^{*} \gamma, \eta\right)_{g}=(\gamma, \partial \eta)_{g}
$$

for $\gamma \in \Lambda^{p+1, q}(M)$ and $\eta \in \Lambda^{p, q}(M)$, where $(\cdot, \cdot)_{g}$ is the $L^{2}$-inner product with respect to $g$. For instance, $(\alpha, \beta)_{g}=\int_{M} g^{\bar{j} i} \alpha_{\bar{j}} \overline{\beta_{\bar{i}}} d V_{g}$ for $(0,1)$-forms $\alpha, \beta$. Analogously, we define the adjoint operator $\bar{\partial}_{g}^{*}$ of $\bar{\partial}$.

Let $(M, g, J)$ be an almost Hermitian manifold with $\operatorname{dim}_{\mathbb{R}} M=2 n$. An affine connection $D$ on $T M$ is called almost Hermitian connection if $D g=D J=0$. For the almost Hermitian connection, we have the following Lemma (cf. $[\mathbf{1}, \mathbf{2}, \mathbf{8}, \mathbf{1 1}])$.

LEMmA 2.1. Let $(M, g, J)$ be an almost Hermitian manifold with $\operatorname{dim}_{\mathbb{R}} M=2 n$. Then for any given vector valued $(1,1)$-form $\Theta=\left(\Theta^{i}\right)_{1 \leq i \leq n}$, there exists a unique almost Hermitian connection $D$ on $(M, g, J)$ such that the $(1,1)$-part of the torsion is equal to the given $\Theta$. 
If the (1, 1)-part of the torsion of an almost Hermitian connection vanishes everywhere, then the connection is called the second canonical connection or the Chern connection. We will refer to the connection as the Chern connection and denote it by $\nabla$.

Now let $\nabla$ be the Chern connection on $M$. We denote the structure coefficients of the Lie bracket by

$$
\begin{aligned}
& {\left[Z_{i}, Z_{j}\right]=: B_{i j}^{r} Z_{r}+B_{i j}^{\bar{r}} Z_{\bar{r}}=B_{i j}^{r} Z_{r}-\overline{N_{\bar{i}}^{r} \bar{j}} Z_{\bar{r}},} \\
& {\left[Z_{i}, Z_{\bar{j}}\right]=: B_{i \bar{j}}^{r} Z_{r}+B_{i \bar{j}}^{\bar{r}} Z_{\bar{r}},} \\
& {\left[Z_{\bar{i}}, Z_{\bar{j}}\right]=: B_{\bar{i} \bar{j}}^{r} Z_{r}+B_{\bar{i} \bar{j}}^{\bar{r}} Z_{\bar{r}}=-N_{\bar{i}-\bar{j}}^{r} Z_{r}+B_{\bar{i} \bar{j}}^{\bar{r}} Z_{\bar{r}},}
\end{aligned}
$$

where we used that $\left[Z_{i}, Z_{j}\right]^{(0,1)}=-\overline{N_{\bar{i} \bar{j}}^{r}} Z_{\bar{r}},\left[Z_{\bar{i}}, Z_{\bar{j}}\right]^{(1,0)}=-N_{\bar{i} \bar{j}}^{r} Z_{r}$ and then $B_{i j}^{\bar{r}}=-\overline{N_{\bar{i} \bar{j}}^{r}}$, $B \bar{i}_{\bar{j}}^{r}=-N_{\bar{i}}^{r}{ }_{\bar{j}}^{r}$. Also we here note that, for instance,

$$
\left[Z_{i}, Z_{\bar{j}}\right]=\left[Z_{i}, Z_{\bar{j}}\right]^{(1,0)}+\left[Z_{i}, Z_{\bar{j}}\right]^{(0,1)},
$$

where

$$
\begin{aligned}
& {\left[Z_{i}, Z_{\bar{j}}\right]^{(1,0)}=\frac{1}{2}\left(\left[Z_{i}, Z_{\bar{j}}\right]-\sqrt{-1} J\left[Z_{i}, Z_{\bar{j}}\right]\right),} \\
& {\left[Z_{i}, Z_{\bar{j}}\right]^{(0,1)}=\frac{1}{2}\left(\left[Z_{i}, Z_{\bar{j}}\right]+\sqrt{-1} J\left[Z_{i}, Z_{\bar{j}}\right]\right) .}
\end{aligned}
$$

Notice that $J$ is integrable if and only if the $B_{i j}^{\bar{r}}$ vanish. We directly compute that

$$
d \zeta^{s}=-\frac{1}{2} B_{k l}^{s} \zeta^{k} \wedge \zeta^{l}-B_{k \bar{l}}^{s} \zeta^{k} \wedge \zeta^{\bar{l}}+\frac{1}{2} N_{\bar{k} \bar{l}}^{s} \zeta^{\bar{k}} \wedge \zeta^{\bar{l}} .
$$

From the computation above, we can split the exterior differential operator $d: \Lambda^{p} M \otimes_{\mathbb{R}} \mathbb{C} \rightarrow$ $\Lambda^{p+1} M \otimes_{\mathbb{R}} \mathbb{C}$ into four components

$$
d=A+\partial+\bar{\partial}+\bar{A}
$$

with

$$
\begin{gathered}
\partial: \Lambda^{p, q} M \rightarrow \Lambda^{p+1, q} M, \quad \bar{\partial}: \Lambda^{p, q} M \rightarrow \Lambda^{p, q+1} M, \\
A: \Lambda^{p, q} M \rightarrow \Lambda^{p+2, q-1} M, \quad \bar{A}: \Lambda^{p, q} M \rightarrow \Lambda^{p-1, q+2} M .
\end{gathered}
$$

In terms of these components, the condition $d^{2}=0$ can be written as

$$
\begin{gathered}
A^{2}=0, \quad \partial A+A \partial=0, \quad \bar{\partial} \bar{A}+\bar{A} \bar{\partial}=0, \quad \bar{A}^{2}=0, \\
A \bar{\partial}+\partial^{2}+\bar{\partial} A=0, \quad A \bar{A}+\partial \bar{\partial}+\bar{\partial} \partial+\bar{A} A=0, \quad \partial \bar{A}+\bar{\partial}^{2}+\bar{A} \partial=0 .
\end{gathered}
$$

Note that for any $p$-form $\psi$, it holds that

$$
\begin{aligned}
d \psi\left(X_{1}, \ldots, X_{p+1}\right)= & \sum_{i=1}^{p+1}(-1)^{i+1} X_{i}\left(\psi\left(X_{1}, \ldots, \widehat{X_{i}}, \ldots, X_{p+1}\right)\right) \\
& +\sum_{i<j}(-1)^{i+j} \psi\left(\left[X_{i}, X_{j}\right], X_{1}, \ldots, \widehat{X_{i}}, \ldots, \widehat{X_{j}}, \ldots, X_{p+1}\right)
\end{aligned}
$$

for any vector fields $X_{1}, \ldots, X_{p+1}$ on $M$ (cf. [11]). 
For any real $(1,1)$-form $\eta=\sqrt{-1} \eta_{i} \zeta^{i} \wedge \zeta^{\bar{j}}$, we have

$$
\begin{aligned}
& \bar{\partial} \eta=\frac{\sqrt{-1}}{2}\left(Z_{\bar{j}}\left(\eta_{k \bar{i}}\right)-Z_{\bar{i}}\left(\eta_{k \bar{j}}\right)-B_{k \bar{i}}^{s} \eta_{s \bar{j}}+B_{k \bar{j}}^{s} \eta_{s \bar{i}}+B_{\bar{i} \bar{j}}^{\bar{s}} \eta_{k \bar{s}}\right) \zeta^{k} \wedge \zeta^{\bar{i}} \wedge \zeta^{\bar{j}}, \\
& \partial \eta=\frac{\sqrt{-1}}{2}\left(Z_{i}\left(\eta_{j \bar{k}}\right)-Z_{j}\left(\eta_{i \bar{k}}\right)-B_{i j}^{s} \eta_{s \bar{k}}-B_{i \bar{k}}^{\bar{s}} \eta_{j \bar{s}}+B_{j \bar{k}}^{\bar{s}} \eta_{i \bar{s}}\right) \zeta^{i} \wedge \zeta^{j} \wedge \zeta^{\bar{k}} .
\end{aligned}
$$

From these computations above, we have

$$
\begin{aligned}
\bar{\partial} \omega & =\frac{\sqrt{-1}}{2}\left(Z_{\bar{j}}\left(g_{k \bar{i}}\right)-Z_{\bar{i}}\left(g_{k \bar{j}}\right)-B_{k \bar{i}}^{s} g_{s \bar{j}}+B_{k \bar{j}}^{s} g_{s \bar{i}}+B_{\bar{i} \bar{j}}^{\bar{s}} g_{k \bar{s}}\right) \zeta^{k} \wedge \zeta^{\bar{i}} \wedge \zeta^{\bar{j}} \\
& =\frac{\sqrt{-1}}{2} T_{\bar{j} \bar{i} k} \zeta^{k} \wedge \zeta^{\bar{i}} \wedge \zeta^{\bar{j}}
\end{aligned}
$$

and

$$
\begin{aligned}
\partial \omega & =\frac{\sqrt{-1}}{2}\left(Z_{i}\left(g_{j \bar{k}}\right)-Z_{j}\left(g_{i \bar{k}}\right)-B_{i j}^{s} g_{s \bar{k}}-B_{i \bar{k}}^{\bar{s}} g_{j \bar{s}}+B_{j \bar{k}}^{\bar{s}} g_{i \bar{s}}\right) \zeta^{i} \wedge \zeta^{j} \wedge \zeta^{\bar{k}} \\
& =\frac{\sqrt{-1}}{2} T_{i j \bar{k}} \zeta^{i} \wedge \zeta^{j} \wedge \zeta^{\bar{k}} .
\end{aligned}
$$

And in this sense, we obtain for any $(0,1)$-form $\beta$,

$$
(\partial \beta)_{k \bar{j}}=Z_{k}\left(\beta_{\bar{j}}\right)-B_{k \bar{j}}^{\bar{m}} \beta_{\bar{m}}=\nabla_{k} \beta_{\bar{j}}, \quad \partial \beta=\nabla_{k} \beta_{\bar{j}} \zeta^{k} \wedge \zeta^{\bar{j}} .
$$

A direct computation yields for any $\varphi \in C^{\infty}(M, \mathbb{R})$,

$$
\begin{aligned}
(d J d \varphi)\left(Z_{i}, Z_{j}\right) & =Z_{i}\left(J d \varphi\left(Z_{j}\right)\right)-Z_{j}\left(J d \varphi\left(Z_{i}\right)\right)-J d \varphi\left(\left[Z_{i}, Z_{j}\right]\right) \\
& =-Z_{i}\left(d \varphi\left(J Z_{j}\right)\right)+Z_{j}\left(d \varphi\left(J Z_{i}\right)\right)+d \varphi\left(J\left[Z_{i}, Z_{j}\right]\right) \\
& =-\sqrt{-1}\left(Z_{i} Z_{j}-Z_{j} Z_{i}\right)(\varphi)+J\left[Z_{i}, Z_{j}\right](\varphi) \\
& =-\sqrt{-1}\left(\left[Z_{i}, Z_{j}\right]+\sqrt{-1} J\left[Z_{i}, Z_{j}\right]\right)(\varphi) \\
& =-2 \sqrt{-1}\left[Z_{i}, Z_{j}\right]^{(0,1)}(\varphi), \\
(d J d \varphi)\left(Z_{\bar{i}}, Z_{\bar{j}}\right) & =Z_{\bar{i}}\left(J d \varphi\left(Z_{\bar{j}}\right)\right)-Z_{\bar{j}}\left(J d \varphi\left(Z_{\bar{i}}\right)\right)-J d \varphi\left(\left[Z_{\bar{i}}, Z_{\bar{j}}\right]\right) \\
& =-Z_{\bar{i}}\left(d \varphi\left(J Z_{\bar{j}}\right)\right)+Z_{\bar{j}}\left(d \varphi\left(J Z_{\bar{i}}\right)\right)+d \varphi\left(J\left[Z_{\bar{i}}, Z_{\bar{j}}\right]\right) \\
& =\sqrt{-1}\left(Z_{\bar{i}} Z_{\bar{j}}-Z_{\bar{j}} Z_{\bar{i}}\right)(\varphi)+J\left[Z_{\bar{i}}, Z_{\bar{j}}\right](\varphi) \\
& =\sqrt{-1}\left(\left[Z_{\bar{i}}, Z_{\bar{j}}\right]-\sqrt{-1} J\left[Z_{\bar{i}}, Z_{\bar{j}}\right]\right)(\varphi) \\
& =2 \sqrt{-1}\left[Z_{\bar{i}}, Z_{\bar{j}}\right]^{(1,0)}(\varphi),
\end{aligned}
$$

and

$$
\begin{aligned}
(d J d \varphi)\left(Z_{i}, Z_{\bar{j}}\right) & =Z_{i}\left(J d \varphi\left(Z_{\bar{j}}\right)\right)-Z_{\bar{j}}\left(J d \varphi\left(Z_{i}\right)\right)-J d \varphi\left(\left[Z_{i}, Z_{\bar{j}}\right]\right) \\
& =-Z_{i}\left(d \varphi\left(J Z_{\bar{j}}\right)\right)+Z_{\bar{j}}\left(d \varphi\left(J Z_{i}\right)\right)+d \varphi\left(J\left[Z_{i}, Z_{\bar{j}}\right]\right) \\
& =\sqrt{-1} Z_{i} Z_{\bar{j}}(\varphi)+\sqrt{-1} Z_{\bar{j}} Z_{i}(\varphi)+J\left[Z_{i}, Z_{\bar{j}}\right](\varphi) \\
& =2 \sqrt{-1} Z_{i} Z_{\bar{j}}(\varphi)-\sqrt{-1}\left(\left[Z_{i}, Z_{\bar{j}}\right]+\sqrt{-1} J\left[Z_{i}, Z_{\bar{j}}\right]\right)(\varphi) \\
& =2 \sqrt{-1}\left(Z_{i} Z_{\bar{j}}-\left[Z_{i}, Z_{\bar{j}}\right]^{(0,1)}\right)(\varphi),
\end{aligned}
$$




$$
\sqrt{-1} \partial \bar{\partial} \varphi=\frac{1}{2}(d J d \varphi)^{(1,1)}=\sqrt{-1}\left(Z_{i} Z_{\bar{j}}-\left[Z_{i}, Z_{\bar{j}}\right]^{(0,1)}\right)(\varphi) \zeta^{i} \wedge \zeta^{\bar{j}}
$$

so we write locally

$$
\partial_{i} \partial_{\bar{j}} \varphi=\left(Z_{i} Z_{\bar{j}}-\left[Z_{i}, Z_{\bar{j}}\right]^{(0,1)}\right) \varphi
$$

\subsection{The torsion and the curvature on almost complex manifolds}

Since the Chern connection $\nabla$ preserves $J$, we are able to define the Christoffel symbols: for $i, j, r=1, \ldots, n$,

$$
\nabla_{i} Z_{j}=\nabla_{Z_{i}} Z_{j}=\Gamma_{i j}^{r} Z_{r}, \quad \nabla_{i} Z_{\bar{j}}=\nabla_{Z_{i}} Z_{\bar{j}}=\Gamma_{i \bar{j}}^{\bar{r}} Z_{\bar{r}},
$$

where

$$
\Gamma_{i j}^{r}=g^{r \bar{s}} Z_{i}\left(g_{j \bar{s}}\right)-g^{r \bar{s}} g_{j \bar{l}} B_{i \bar{s}}^{\bar{l}}, \quad \Gamma_{i p}^{p}=Z_{i}(\log \operatorname{det} g)-B_{i \bar{s}}^{\bar{s}} .
$$

For any $(0,1)$-form $\beta$, we have $\beta=\beta_{\bar{j}} \zeta^{\bar{j}}$,

$$
\nabla_{k} \beta_{\bar{j}}=Z_{k}\left(\beta_{\bar{j}}\right)-\Gamma_{k \bar{j}}^{\bar{l}} \beta_{\bar{l}}=Z_{k}\left(\beta_{\bar{j}}\right)-B_{k \bar{j}}^{\bar{l}} \beta_{\bar{l}} .
$$

Note that the mixed derivatives $\nabla_{i} Z_{\bar{j}}$ do not depend on $g$.

The torsion $T=\left(T^{i}\right)$ of the Chern connection $\nabla$ is defined by

$$
T^{i}:=d \zeta^{i}-\zeta^{p} \wedge \omega_{p}^{i}, \quad T^{\bar{i}}:=d \zeta^{\bar{i}}-\zeta^{\bar{p}} \wedge \omega_{\bar{p}}^{\bar{i}},
$$

where $\omega=\left(\omega_{j}^{i}\right)$ is the connection form defined by $\omega_{j}^{i}:=\Gamma_{k j}^{i} \zeta^{k}+\Gamma_{\bar{k} j}^{i} \zeta^{\bar{k}}$. Since the torsion $T$ of the Chern connection $\nabla$ has no $(1,1)$-part,

$$
\begin{aligned}
0=T_{k \bar{l}}^{i} & =T^{i}\left(Z_{k}, Z_{\bar{l}}\right) \\
& =-\zeta^{i}\left(\left[Z_{k}, Z_{\bar{l}}\right]\right)-\left(\Gamma_{s p}^{i} \zeta^{p} \wedge \zeta^{s}+\Gamma_{\bar{s} p}^{i} \zeta^{p} \wedge \zeta^{\bar{s}}\right)\left(Z_{k}, Z_{\bar{l}}\right) \\
& =-B_{k \bar{l}}^{i}-\Gamma_{\bar{l} l}^{i} \\
& =B_{\bar{l} k}^{i}-\Gamma_{\bar{l} k}^{i}, \\
0=T_{k \bar{l}}^{\bar{i}} & =T^{\bar{i}}\left(Z_{k}, Z_{\bar{l}}\right) \\
& =-\zeta^{\bar{i}}\left(\left[Z_{k}, Z_{\bar{l}}\right]\right)-\left(\Gamma_{s \bar{p}}^{\bar{i}} \zeta^{\bar{p}} \wedge \zeta^{s}+\Gamma_{\bar{s} \bar{p}}^{\bar{i}} \zeta^{\bar{p}} \wedge \zeta^{\bar{s}}\right)\left(Z_{k}, Z_{\bar{l}}\right) \\
& =-B_{k \bar{l}}^{\bar{i}}+\Gamma_{k \bar{l}}^{\bar{i}},
\end{aligned}
$$

we have

$$
\Gamma_{i \bar{j}}^{\bar{r}}=B_{i \bar{j}}^{\bar{r}}
$$

Here note that the $B_{j \bar{b}}^{\bar{q}}$ and $B_{\bar{j} b}^{q}$ do not depend on $g$, and depend only on $J$ since the mixed derivatives $\nabla_{j} Z_{\bar{b}}$ do not depend on $g$. 
The torsion $T$ of $\nabla$ has no $(1,1)$-part and the only non-vanishing components are as follows:

$$
\begin{aligned}
T_{i j}^{s} & =T^{s}\left(Z_{i}, Z_{j}\right) \\
& =-\zeta^{s}\left(\left[Z_{i}, Z_{j}\right]\right)-\left(\Gamma_{s p}^{i} \zeta^{p} \wedge \zeta^{s}+\Gamma_{\bar{s} p}^{i} \zeta^{p} \wedge \zeta^{\bar{s}}\right)\left(Z_{i}, Z_{j}\right) \\
& =-B_{i j}^{s}-\Gamma_{j i}^{s}+\Gamma_{i j}^{s} \\
T_{i j}^{\bar{s}} & =d \zeta^{\bar{s}}\left(Z_{i}, Z_{j}\right)=-\zeta^{\bar{s}}\left(\left[Z_{i}, Z_{j}\right]\right)=-B_{i j}^{\bar{s}} .
\end{aligned}
$$

On the other hand we have $d \zeta^{\bar{s}}\left(Z_{i}, Z_{j}\right)=N_{i j}^{\bar{s}}$, hence we obtain that $N_{i j}^{\bar{s}}=-B_{i j}^{\bar{s}}$. These computations tell us that $T$ splits in $T=T^{\prime}+T^{\prime \prime}$, where $T^{\prime} \in \Gamma\left(\Lambda^{2,0}(M) \otimes T^{1,0} M\right)$, a section of $\Lambda^{2,0}(M) \otimes T^{1,0} M$, and $T^{\prime \prime} \in \Gamma\left(\Lambda^{2,0}(M) \otimes T^{0,1} M\right)$. The torsion $T=\left(T^{i}\right)$ can be split into $T=T^{2,0}+T^{1,1}+T^{0,2}=T^{2,0}+T^{0,2}$ since $T^{1,1}=0$, where $T^{2,0}=\left(\frac{1}{2} T_{j k}^{i} \zeta^{j} \wedge\right.$ $\left.\zeta^{k}\right)_{1 \leq i \leq n}, T^{0,2}=\left(\frac{1}{2} N_{\bar{j} \bar{k}}^{i} \zeta^{\bar{j}} \wedge \zeta^{\bar{k}}\right)_{1 \leq i \leq n}$, which tells us that the $(0,2)$-part of the Chern connection is uniquely determined by the Nijenhuis tensor (cf. [11]).

We also lower the index of torsion and denote it by

$$
T_{i j \bar{k}}=T_{i j}^{s} g_{s \bar{k}}=Z_{i}\left(g_{j \bar{k}}\right)-Z_{j}\left(g_{i \bar{k}}\right)+B_{\bar{k} i}^{\bar{q}} g_{j \bar{q}}-B_{\bar{k} j}^{\bar{q}} g_{i \bar{q}}-B_{i j}^{s} g_{s \bar{k}} .
$$

Note that $T^{\prime \prime}$ depends only on $J$ and it can be regarded as the Nijenhuis tensor of $J$, that is, $J$ is integrable if and only if $T^{\prime \prime}$ vanishes.

We denote by $\Omega$ the curvature of the Chern connection $\nabla$. We can regard $\Omega$ as a section of $\Lambda^{2}(M) \otimes T M, \Omega \in \Gamma\left(\Lambda^{2}(M) \otimes T M\right)$ and $\Omega$ splits in $\Omega=H+R+\bar{H}$, where $R \in \Gamma\left(\Lambda^{1,1}(M) \otimes \Lambda^{1,1}(M)\right), H \in \Gamma\left(\Lambda^{2,0}(M) \otimes \Lambda^{1,1}(M)\right)$.

The curvature form can be written as $\Omega_{j}^{i}=d \omega_{j}^{i}+\omega_{s}^{i} \wedge \omega_{j}^{s}$.

In terms of the $Z_{r}$, we have

$$
\begin{aligned}
& R_{i \bar{j} k}^{r}=\Omega_{k}^{r}\left(Z_{i}, Z_{\bar{j}}\right)=Z_{i}\left(\Gamma_{\bar{j} k}^{r}\right)-Z_{\bar{j}}\left(\Gamma_{i k}^{r}\right)+\Gamma_{i s}^{r} \Gamma_{\bar{j} k}^{s}-\Gamma_{\bar{j} s}^{r} \Gamma_{i k}^{s}-B_{i \bar{j}}^{s} \Gamma_{s k}^{r}+B_{\bar{j} i}^{\bar{s}} \Gamma_{\bar{s} k}^{r}, \\
& H_{i j k}^{r}=\Omega_{k}^{r}\left(Z_{i}, Z_{j}\right)=Z_{i}\left(\Gamma_{j k}^{r}\right)-Z_{j}\left(\Gamma_{i k}^{r}\right)+\Gamma_{i s}^{r} \Gamma_{j k}^{s}-\Gamma_{j s}^{r} \Gamma_{i k}^{s}-B_{i j}^{s} \Gamma_{s k}^{r}-B_{i j}^{\bar{s}} \Gamma_{\bar{s} k}^{r}, \\
& H_{\bar{i} \bar{j} k}^{r}=\Omega_{k}^{r}\left(Z_{\bar{i}}, Z_{\bar{j}}\right)=Z_{\bar{i}}\left(\Gamma_{\bar{j} k}^{r}\right)-Z_{\bar{j}}\left(\Gamma_{\bar{i} k}^{r}\right)+\Gamma_{\bar{i} s}^{r} \Gamma_{\bar{j} k}^{s}-\Gamma_{\bar{j} s}^{r} \Gamma_{\bar{i} k}^{s}-B_{\bar{i} \bar{j}}^{s} \Gamma_{s k}^{r}-B_{\bar{i} \bar{j}}^{\bar{s}} \Gamma_{\bar{s} k}^{r},
\end{aligned}
$$

and we deduce that, using $\Gamma_{k p}^{p}=Z_{k}(\log \operatorname{det} g)-B_{k \bar{p}}^{\bar{p}}$,

$$
\begin{aligned}
P_{i \bar{j}}= & R_{i \bar{j} r}^{r} \\
= & Z_{i}\left(\Gamma_{\bar{j} r}^{r}\right)-Z_{\bar{j}}\left(\Gamma_{i r}^{r}\right)-B_{i \bar{j}}^{s} \Gamma_{s r}^{r}-B_{i \bar{j}}^{\bar{s}} \Gamma_{\bar{s} r}^{r} \\
= & Z_{i}\left(\Gamma_{\bar{j} r}^{r}\right)-Z_{\bar{j}} Z_{i}(\log \operatorname{det} g)+Z_{\bar{j}}\left(B_{i \bar{r}}^{\bar{r}}\right)-B_{i \bar{j}}^{s} Z_{s}(\log \operatorname{det} g)+B_{i \bar{j}}^{s} B_{s \bar{r}}^{\bar{r}}-B_{i \bar{j}}^{\bar{s}} \Gamma_{\bar{s} r}^{r} \\
= & Z_{i}\left(\Gamma_{\bar{j} r}^{r}\right)+\left[Z_{i}, Z_{\bar{j}}\right](\log \operatorname{det} g)-Z_{i} Z_{\bar{j}}(\log \operatorname{det} g)+Z_{\bar{j}}\left(B_{i \bar{r}}^{\bar{r}}\right) \\
& \quad-\left[Z_{i}, Z_{\bar{j}}^{(1,0)}(\log \operatorname{det} g)+B_{i \bar{j}}^{s} B_{s \bar{r}}^{\bar{r}}-B_{i \bar{j}}^{\bar{s}} \Gamma_{\bar{s} r}^{r}\right. \\
= & -\left(Z_{i} Z_{\bar{j}}-\left[Z_{i}, Z_{\bar{j}}\right]^{(0,1)}\right)(\log \operatorname{det} g)+Z_{\bar{j}}\left(B_{i \bar{r}}^{\bar{r}}\right)+Z_{i}\left(B_{\bar{j} r}^{r}\right)+B_{i \bar{j}}^{s} B_{s \bar{r}}^{\bar{r}}-B_{i \bar{j}}^{\bar{s}} B_{\bar{s} r}^{r}
\end{aligned}
$$




$$
\begin{aligned}
R_{i j}= & H_{i j r}{ }^{r} \\
= & Z_{i}\left(\Gamma_{j k}^{r}\right)-Z_{j}\left(\Gamma_{i k}^{r}\right)-B_{i j}^{s} \Gamma_{s k}^{r}-B_{i j}^{\bar{s}} \Gamma_{\bar{s} k}^{r} \\
= & Z_{i} Z_{j}(\log \operatorname{det} g)-Z_{i}\left(B_{j \bar{r}}^{\bar{r}}\right)-Z_{j} Z_{i}(\log \operatorname{det} g)+Z_{j}\left(B_{i \bar{r}}^{\bar{r}}\right) \\
& -B_{i j}^{s} Z_{s}(\log \operatorname{det} g)+B_{i j}^{s} B_{s \bar{r}}^{\bar{r}}-B_{i j}^{\bar{s}} B_{\bar{s} r}^{r} \\
= & \left(\left[Z_{i}, Z_{j}\right]-\left[Z_{i}, Z_{j}\right]^{(1,0)}\right)(\log \operatorname{det} g)-Z_{i}\left(B_{j \bar{r}}^{\bar{r}}\right)+Z_{j}\left(B_{i \bar{r}}^{\bar{r}}\right)+B_{i j}^{s} B_{s \bar{r}}^{\bar{r}}-B_{i j}^{\bar{s}} B_{\bar{s} r}^{r} \\
= & {\left[Z_{i}, Z_{j}\right]^{(0,1)}(\log \operatorname{det} g)-Z_{i}\left(B_{j \bar{r}}^{\bar{r}}\right)+Z_{j}\left(B_{i \bar{r}}^{\bar{r}}\right)+B_{i j}^{s} B_{s \bar{r}}^{\bar{r}}-B_{i j}^{\bar{s}} B_{\bar{s} r}^{r} }
\end{aligned}
$$

and

$$
\begin{aligned}
R_{\bar{i} \bar{j}} & =H_{\bar{i} \bar{j} r}^{r} \\
& =Z_{\bar{i}}\left(\Gamma_{\bar{j} r}^{r}\right)-Z_{\bar{j}}\left(\Gamma_{\bar{i} r}^{r}\right)-B_{\bar{i} \bar{j}}^{s} \Gamma_{s r}^{r}-B_{\bar{i} \bar{j}}^{\bar{s}} \Gamma_{\bar{s} r}^{r} \\
& =Z_{\bar{i}}\left(B_{\bar{j} r}^{r}\right)-Z_{\bar{j}}\left(B_{\bar{i} r}^{r}\right)-B_{\bar{i} \bar{j}}^{s} Z_{s}(\log \operatorname{det} g)+B_{\bar{i} \bar{j}}^{s} B_{s \bar{r}}^{\bar{r}}-B_{\bar{i} \bar{j}}^{\bar{s}} B_{\bar{s} r}^{r} \\
& =-\left[Z_{\bar{i}}^{r}, Z_{\bar{j}}\right]^{(1,0)}(\log \operatorname{det} g)+Z_{\bar{i}}\left(B_{\bar{j} r}^{r}\right)-Z_{\bar{j}}\left(B_{\bar{i} r}^{r}\right)+B_{\bar{i} \bar{j}}^{s} B_{s \bar{r}}^{\bar{r}}-B_{\bar{i} \bar{j}}^{\bar{s}} B_{\bar{s} r}^{r} .
\end{aligned}
$$

Let $(M, g, J)$ be an almost Hermitian manifold. Let $\left\{Z_{r}\right\}$ be a local unitary $(1,0)$-frame with respect to $g$ around a fixed point $p \in M$. Note that unitary frames always exist locally since we can take any frame and apply the Gram-Schmidt process. Then with respect to such a frame, we have $g_{i} \bar{j}=\delta_{i j}, Z_{k}\left(g_{i} \bar{j}\right)=0$.

LEMMA 2.2. Using a local g-unitary (1,0)-frame, the Christoffel symbols satisfy

$$
\Gamma_{i j}^{k}=-\Gamma_{i \bar{k}}^{\bar{j}}
$$

Proof. We compute with a local $g$-unitary $(1,0)$-frame,

$$
\Gamma_{i j}^{k}=g\left(\nabla_{i} Z_{j}, Z_{\bar{k}}\right)=Z_{i}\left(g_{j \bar{k}}\right)-g\left(Z_{j}, \nabla_{i} Z_{\bar{k}}\right)=-\Gamma_{i \bar{k}}^{\bar{j}} .
$$

With a local $g$-unitary $(1,0)$-frame, the components of the torsion can be written as

$$
T_{i j}^{k}=-B_{i \bar{k}}^{\bar{j}}+B_{j \bar{k}}^{\bar{i}}-B_{i j}^{k}
$$

and the components of $w$ can be written as

$$
w_{j}=-B_{j r}^{r}-B_{j \bar{r}}^{\bar{r}}+B_{r \bar{r}}^{\bar{j}} .
$$

Using a local $g$-unitary $(1,0)$-frame $\left\{Z_{r}\right\}$, we obtain the following computation:

$$
\begin{aligned}
g( & \left.\nabla_{i} \nabla_{\bar{j}} Z_{k}-\nabla_{\bar{j}} \nabla_{i} Z_{k}-\nabla_{\left[Z_{i}, Z_{\bar{j}}\right]} Z_{k}, Z_{\bar{l}}\right) \\
= & Z_{i}\left(g\left(\nabla_{\bar{j}} Z_{k}, Z_{\bar{l}}\right)\right)-g\left(\nabla_{\bar{j}} Z_{k}, \nabla_{i} Z_{\bar{l}}\right)-Z_{\bar{j}}\left(g\left(\nabla_{i} Z_{k}, Z_{\bar{l}}\right)\right)+g\left(\nabla_{i} Z_{k}, \nabla_{\bar{j}} Z_{\bar{l}}\right) \\
& \quad-B_{i \bar{j}}^{r} g\left(\nabla_{r} Z_{k}, Z_{\bar{l}}\right)-B_{i \bar{j}}^{\bar{r}} g\left(\nabla_{\bar{r}} Z_{k}, Z_{\bar{l}}\right) \\
= & Z_{i}\left(\Gamma_{\bar{j} k}^{s} g_{s \bar{l}}\right)-\Gamma_{\bar{j} k}^{s} \Gamma_{i \bar{l}}^{\bar{r}} g_{s \bar{r}}-Z_{\bar{j}}\left(\Gamma_{i k}^{s} g_{s \bar{l}}\right)+\Gamma_{i k}^{s} \Gamma_{\bar{j} \bar{l}}^{\bar{r}} g_{s \bar{r}}-B_{i \bar{j}}^{s} \Gamma_{s k}^{r} g_{r \bar{l}}-B_{i \bar{j}}^{\bar{r}} \Gamma_{\bar{r} k}^{s} g_{s \bar{l}} \\
= & Z_{i}\left(\Gamma_{\bar{j} k}^{l}\right)-Z_{\bar{j}}\left(\Gamma_{i k}^{l}\right)+\Gamma_{i s}^{l} \Gamma_{\bar{j} k}^{s}-\Gamma_{\bar{j} s}^{l} \Gamma_{i k}^{s}-B_{i \bar{j}}^{s} \Gamma_{s k}^{l}-B_{i \bar{j}}^{\bar{s}_{\bar{s} k}} \Gamma_{\bar{s} k}^{l} \\
= & R_{i \bar{j} k}^{s} g_{s \bar{l}} \\
= & R_{i \bar{j} k \bar{l}} .
\end{aligned}
$$


Therefore we obtain the following formula.

LEMMA 2.3. One has

$$
R_{i \bar{j} k \bar{l}}=g\left(\nabla_{i} \nabla_{\bar{j}} Z_{k}-\nabla_{\bar{j}} \nabla_{i} Z_{k}-\nabla_{\left[Z_{i}, Z_{\bar{j}}\right]} Z_{k}, Z_{\bar{l}}\right) .
$$

The Chern-Ricci form $\operatorname{Ric}(\omega)$ is defined by

$$
\operatorname{Ric}(\omega):=\frac{\sqrt{-1}}{2} R_{k l} \zeta^{k} \wedge \zeta^{l}+\sqrt{-1} P_{k \bar{l}} \zeta^{k} \wedge \zeta^{\bar{l}}+\frac{\sqrt{-1}}{2} R_{\bar{k} \bar{l}} \zeta^{\bar{k}} \wedge \zeta^{\bar{l}}
$$

It is a closed real 2-form. If $J$ is integrable, it is a closed real $(1,1)$-form. If, furthermore, $J$ is integrable and $d \omega=0$, then the Chern-Ricci form coincides with the Ricci form defined by the Levi-Civita connection of $\omega$.

Lemma 2.4. (The first Bianchi identity for the Chern curvature) For any $X, Y, Z \in T^{\mathbb{C}} M$,

$$
\sum \Omega(X, Y) Z=\sum\left(T(T(X, Y), Z)+\nabla_{X} T(Y, Z)\right),
$$

where the sum is taken over all cyclic permutations.

This identity induces the following formulae:

$$
\begin{gathered}
R_{i \bar{j} k}{ }^{l}=R_{k \bar{j} i}{ }^{l}-T_{i k}^{\bar{r}} T_{\bar{r} \bar{j}}^{l}+\nabla_{\bar{j}} T_{k i}^{l}=R_{k \bar{j} i}{ }^{l}-B_{i k}^{\bar{r}} B_{\bar{r} \bar{j}}^{l}+\nabla_{\bar{j}} T_{k i}^{l}, \\
H_{i j k}{ }^{l}=T_{j i}^{\bar{r}} T_{\bar{r} \bar{l}}^{\bar{k}}+\nabla_{\bar{l}} T_{j i}^{\bar{k}}=-B_{j i}^{\bar{r}} T_{\bar{r} \bar{l}}^{\bar{k}}-\nabla_{\bar{l}} B_{j i}^{\bar{k}} .
\end{gathered}
$$

Taking into account the Bianchi identities, we have the following lemma.

LEMMA 2.5. [8, Lemma 3.5] The formula

$$
P=S+\operatorname{div}^{\nabla} T^{\prime}-\nabla \bar{w}+Q^{7}+Q^{8}
$$

holds for any almost Hermitian metric $g$, where $T^{\prime}$ is the torsion of the Chern connection $\nabla$ associated to $g$,

$$
\left(\operatorname{div}^{\nabla} T^{\prime}\right)_{i \bar{j}}=g^{k \bar{l}} \nabla_{\bar{l}} T_{k i \bar{j}}, \quad(\nabla \bar{w})_{i \bar{j}}=g^{k \bar{l}} \nabla_{i} T_{\bar{j} \bar{l} k} .
$$

\section{The short-time existence and the uniqueness}

In this section, we will prove Theorem 1.1 and Theorem 1.2. Let $\left(M^{2 n}, g, J\right)$ be a compact almost Hermitian manifold.

We need the following lemma.

LeMma 3.1. [8, Lemma 4.1] Let $(M, g, J)$ be a compact almost Hermitian manifold. Let $\alpha$ be a $(0,1)$-form and $\left\{Z_{j}\right\}$ be an arbitrary unitary $(1,0)$-frame. Then we have

$$
\int_{M} Z_{r}\left(\alpha_{\bar{r}}\right) d V_{g}=\int_{M} \alpha_{\bar{i}}\left(B_{i r}^{r}+B_{i \bar{r}}^{\bar{r}}\right) d V_{g},
$$

where $d V_{g}=(1 / n !) \omega^{n}$. 
Let $\left\{Z_{r}\right\}$ be an arbitrary local unitary $(1,0)$-frame with respect to $g$ and let $\left\{\zeta^{r}\right\}$ be the associated coframe. Then $\omega$ takes the expression $\omega=\sqrt{-1} \delta_{r \bar{k}} \zeta^{r} \wedge \zeta^{\bar{k}}$ and we can write $\alpha=\alpha_{\bar{r}} \zeta^{\bar{r}}$. Note that as we see from (๒) in Section 2, we have

$$
\begin{aligned}
\partial \alpha & =(\partial \alpha)_{r \bar{k}} \zeta^{r} \wedge \zeta^{\bar{k}} \\
& =\nabla_{r} \alpha_{\bar{k}} \zeta^{r} \wedge \zeta^{\bar{k}} \\
& =\left(Z_{r}\left(\alpha_{\bar{k}}\right)-\Gamma_{r \bar{k}}^{\bar{m}} \alpha_{\bar{m}}\right) \zeta^{r} \wedge \zeta^{\bar{k}} \\
& =\left(Z_{r}\left(\alpha_{\bar{k}}\right)-B_{r \bar{k}}^{\bar{m}} \alpha_{\bar{m}}\right) \zeta^{r} \wedge \zeta^{\bar{k}} .
\end{aligned}
$$

Applying the result of Lemma 3.1, we obtain the following formulae.

LEMMA 3.2. Given an almost Hermitian metric $\omega$, we have

$$
\left(\partial_{g}^{*} \omega\right)_{\bar{j}}=-\sqrt{-1} w_{\bar{j}}, \quad\left(\bar{\partial}_{g}^{*} \omega\right)_{k}=-\sqrt{-1} w_{k} .
$$

Proof. Let $\left\{Z_{r}\right\}$ be an arbitrary local $g$-unitary frame. For any $\alpha \in \Lambda^{0,1}$, we compute

$$
\begin{aligned}
\left(\partial_{g}^{*} \omega, \alpha\right)_{g} & =(\omega, \partial \alpha)_{g} \\
& =\int_{M} g^{i \bar{j}} g^{k \bar{l}} \omega_{i \bar{l}} \overline{(\partial \alpha)_{j \bar{k}}} d V_{g} \\
& =\sqrt{-1} \int_{M} g^{k \bar{j}} \overline{(\partial \alpha)_{j \bar{k}}} d V_{g} \\
& =\sqrt{-1} \int_{M} \overline{(\partial \alpha)_{j \bar{j}}} d V_{g} \\
& =\sqrt{-1} \int_{M} \overline{\nabla_{j} \alpha_{\bar{j}}} d V_{g} \\
& =\sqrt{-1} \int_{M} \overline{\left(Z_{j}\left(\alpha_{\bar{j}}\right)-B_{j \bar{j}}^{\bar{r}} \alpha_{\bar{r}}\right)} d V_{g} \\
& =\sqrt{-1} \int_{M} \overline{\left(B_{j r}^{r}+B_{j \bar{r}}^{\bar{r}}-B_{r \bar{r}}^{\bar{j}}\right.} \overline{\alpha_{\bar{j}}} d V_{g} \\
& =-\sqrt{-1} \int_{M} w_{\bar{j}} \overline{\alpha_{\bar{j}}} d V_{g},
\end{aligned}
$$

where we used the result in Lemma 3.1 in the fourth line. This gives the first formula, and we obtain that $\partial_{g}^{*} \omega=-\sqrt{-1} w_{\bar{j}} \zeta^{\bar{j}}$. The second one follows analogously.

LEMMA 3.3. Given an almost Hermitian metric $\omega$, we have

$$
\left(\partial \partial_{g}^{*} \omega\right)_{i \bar{j}}=-\sqrt{-1} \nabla_{i} w_{\bar{j}}, \quad\left(\bar{\partial} \bar{\partial}_{g}^{*} \omega\right)_{k \bar{l}}=-\sqrt{-1} \nabla_{\bar{l}} w_{k} .
$$

Proof. In general, we have, for any $\alpha \in \Lambda^{0,1},(\partial \alpha)_{i} \bar{j}=\nabla_{i} \alpha_{\bar{j}}$. Hence we have

$$
\begin{aligned}
\left(\partial \partial_{g}^{*} \omega\right)_{i \bar{j}} & =-\sqrt{-1}(\partial w)_{i \bar{j}} \\
& =-\sqrt{-1} \nabla_{i} w_{\bar{j}},
\end{aligned}
$$

and we obtain that $\partial \partial_{g}^{*} \omega=-\sqrt{-1} \nabla_{i} w_{\bar{j}} \zeta^{i} \wedge \zeta^{\bar{j}}$. The second follows analogously. 
Now we consider the operator

$$
\Phi(\omega)=-\partial \partial_{g}^{*} \omega-\bar{\partial} \bar{\partial}_{g}^{*} \omega+P(\omega) .
$$

PROPOSITION 3.1. The operator $\omega \mapsto \Phi(\omega)$ is a nonlinear second-order elliptic operator for an almost Hermitian metric $\omega$.

Proof. Let $\left\{Z_{r}\right\}$ be an arbitrary local $(1,0)$-frame with respect to $J$. We first compute

$$
\begin{aligned}
\left(\partial \partial_{g}^{*} \omega\right)_{i \bar{j}}+\left(\bar{\partial} \bar{\partial}_{g}^{*} \omega\right)_{i \bar{j}} & =-\sqrt{-1} \nabla_{i} w_{\bar{j}}-\sqrt{-1} \nabla_{\bar{j}} w_{i} \\
& =\sqrt{-1}\left\{-\left(Z_{i}\left(w_{\bar{j}}\right)-\Gamma_{i \bar{j}}^{\bar{r}} w_{\bar{r}}\right)-\left(Z_{\bar{j}}\left(w_{i}\right)-\Gamma_{\bar{j} i}^{r} w_{r}\right)\right\} \\
& =\sqrt{-1}\left\{-Z_{i}\left(w_{\bar{j}}\right)+B_{i \bar{j}}^{\bar{r}} w_{\bar{r}}-Z_{\bar{j}}\left(w_{i}\right)+B_{\bar{j} i}^{r} w_{r}\right\} .
\end{aligned}
$$

Now let $D$ be the Levi-Civita connection with respect to $g$ and let $\nabla$ be the Chern connection with respect to $g$. Set $A:=D-\nabla$. The relation between $D$ and $\nabla$ is as follows (cf. [1, Lemma 2.2], [9], [10, Lemma 3.1]):

$$
g\left(D_{Y} X, Z\right)=g\left(\nabla_{Y} X, Z\right)+\frac{1}{2}(g(T(X, Y), Z)+g(T(Y, Z), X)-g(T(Z, X), Y))
$$

for any tangent vector fields $X, Y$ and $Z$. Here notice that the torsion $T$ of the Chern connection $\nabla$ is also defined as

$$
T(X, Y)=\nabla_{X} Y-\nabla_{Y} X-[X, Y],
$$

and in this sense, we compute as follows with a local $(1,0)$-frame $\left\{Z_{r}\right\}$ :

$$
\begin{aligned}
T_{i j}:=T\left(Z_{i}, Z_{j}\right) & =\nabla_{Z_{i}} Z_{j}-\nabla_{Z_{j}} Z_{i}-\left[Z_{i}, Z_{j}\right] \\
& =\Gamma_{i j}^{k} Z_{k}-\Gamma_{j i}^{k} Z_{k}-B_{i j}^{k} Z_{k}-B_{i j}^{\bar{k}} Z_{\bar{k}}=T_{i j}^{k} Z_{k}+T_{i j}^{\bar{k}} Z_{\bar{k}}, \\
T_{i \bar{j}}=T\left(Z_{i}, Z_{\bar{j}}\right) & =\nabla_{Z_{i}} Z_{\bar{j}}-\nabla_{Z_{\bar{j}}} Z_{i}-\left[Z_{i}, Z_{\bar{j}}\right] \\
& =\left(B_{\bar{j} i}^{k}-\Gamma_{\bar{j} i}^{k}\right) Z_{k}+\left(\Gamma_{i \bar{j}}^{\bar{k}}-B_{i \bar{j}}^{\bar{k}}\right) Z_{\bar{k}}=0 .
\end{aligned}
$$

Then we have

$$
\begin{aligned}
g\left(A_{i} Z_{j}-A_{j} Z_{i}, Z_{\bar{s}}\right)= & \frac{1}{2}\left(g\left(T_{j i}, Z_{\bar{s}}\right)+g\left(T_{i \bar{s}}, Z_{j}\right)-g\left(T_{\bar{s} j}, Z_{i}\right)\right) \\
& -\frac{1}{2}\left(g\left(T_{i j}, Z_{\bar{s}}\right)+g\left(T_{j \bar{s}}, Z_{i}\right)-g\left(T_{\bar{s} i}, Z_{j}\right)\right) \\
= & g\left(T_{j i}, Z_{\bar{s}}\right) \\
= & g\left(T_{j i}^{k} Z_{k}+T_{j i}^{\bar{k}} Z_{\bar{k}}, Z_{\bar{s}}\right) .
\end{aligned}
$$

Hence we obtain

$$
\begin{aligned}
T_{j i \bar{s}} & =g\left(T_{j i}^{k} Z_{k}, Z_{\bar{s}}\right) \\
& =g\left(A_{i} Z_{j}-A_{j} Z_{i}-T_{j i}^{\bar{k}} Z_{\bar{k}}, Z_{\bar{s}}\right) \\
& =g\left(A_{i} Z_{j}, Z_{\bar{s}}\right)-g\left(A_{j} Z_{i}, Z_{\bar{s}}\right) \\
& =-g\left(Z_{j}, A_{i} Z_{\bar{s}}\right)+g\left(Z_{i}, A_{j} Z_{\bar{s}}\right) \\
& =B_{i \bar{s}}^{\bar{k}} g_{j \bar{k}}-B_{j \bar{s}}^{\bar{k}} g_{i \bar{k}}-g\left(Z_{j}, D_{i} Z_{\bar{s}}\right)+g\left(Z_{i}, D_{j} Z_{\bar{s}}\right),
\end{aligned}
$$


where we used that

$$
0=Z_{i}\left(g_{k \bar{l}}\right)-Z_{i}\left(g_{k \bar{l}}\right)=A_{i}\left(g_{k \bar{l}}\right)=g\left(A_{i} Z_{k}, Z_{\bar{l}}\right)+g\left(Z_{k}, A_{i} Z_{\bar{l}}\right) .
$$

Similarly we have

$$
\begin{aligned}
T_{\bar{j} \bar{r} r} & =B_{\bar{r} r}^{k} g_{k \bar{j}}-B_{\bar{j} r}^{k} g_{k \bar{r}}-g\left(Z_{\bar{j}}, D_{\bar{r}} Z_{r}\right)+g\left(Z_{\bar{r}}, D_{\bar{j}} Z_{r}\right), \\
T_{i r \bar{r}} & =B_{r \bar{r}}^{\bar{k}} g_{i \bar{k}}-B_{i \bar{r}}^{\bar{k}} g_{r \bar{k}}-g\left(Z_{i}, D_{r} Z_{\bar{r}}\right)+g\left(Z_{r}, D_{j} Z_{\bar{r}}\right) .
\end{aligned}
$$

Using these formulae, we compute

$$
\begin{aligned}
-Z_{\bar{j}}\left(w_{i}\right)=- & Z_{\bar{j}}\left(T_{i r \bar{r}}\right) \\
=- & Z_{\bar{j}}\left(g\left(Z_{r}, D_{i} Z_{\bar{r}}\right)\right)+Z_{\bar{j}}\left(g\left(Z_{i}, D_{r} Z_{\bar{r}}\right)\right) \\
& \quad-Z_{\bar{j}}\left(B_{r \bar{r}}^{\bar{k}}\right) g_{i \bar{k}}+Z_{\bar{j}}\left(B_{i \bar{r}}^{\bar{k}}\right) g_{r \bar{k}}-B_{r \bar{r}}^{\bar{k}} Z_{\bar{j}}\left(g_{i \bar{k}}\right)+B_{i \bar{r}}^{\bar{k}} Z_{\bar{j}}\left(g_{r \bar{k}}\right), \\
-Z_{i}\left(w_{\bar{j}}\right)=- & Z_{i}\left(T_{\bar{j} \bar{r} r}\right) \\
=- & Z_{i}\left(g\left(Z_{\bar{r}}, D_{\bar{j}} Z_{r}\right)\right)+Z_{i}\left(g\left(Z_{\bar{j}}, D_{\bar{r}} Z_{r}\right)\right)-Z_{i}\left(B_{\bar{r} r}^{k}\right) g_{k \bar{j}}+Z_{i}\left(B_{\bar{j} r}^{k}\right) g_{k \bar{r}} \\
& -B_{\bar{r} r}^{k} Z_{i}\left(g_{k \bar{j}}\right)+B_{\bar{j} r}^{k} Z_{i}\left(g_{k \bar{r}}\right) \\
= & -Z_{i}\left(g\left(Z_{\bar{r}}, D_{\bar{j}} Z_{r}\right)\right)+Z_{i}\left(g\left(Z_{\bar{j}},\left[Z_{\bar{r}}, Z_{r}\right]\right)\right)+Z_{i}\left(g\left(Z_{\bar{j}}, D_{r} Z_{\bar{r}}\right)\right) \\
& -Z_{i}\left(B_{\bar{r} r}^{k}\right) g_{k \bar{j}}+Z_{i}\left(B_{\bar{j} r}^{k}\right) g_{k \bar{r}}-B_{\bar{r} r}^{k} Z_{i}\left(g_{k \bar{j}}\right)+B_{\bar{j} r}^{k} Z_{i}\left(g_{k \bar{r}}\right) .
\end{aligned}
$$

Since we would like to know how much the terms $\partial \partial_{g}^{*} \omega+\bar{\partial} \bar{\partial}_{g}^{*} \omega$ involve the second derivatives of $g$, we calculate $-Z_{\bar{j}}\left(w_{i}\right)-Z_{i}\left(w_{\bar{j}}\right)$ specifically as follows:

$$
\begin{aligned}
- & Z_{i}\left(g\left(Z_{\bar{r}}, D_{\bar{j}} Z_{r}\right)\right)-Z_{\bar{j}}\left(g\left(Z_{r}, D_{i} Z_{\bar{r}}\right)\right) \\
& =-g\left(D_{i} Z_{\bar{r}}, D_{\bar{j}} Z_{r}\right)-g\left(Z_{\bar{r}}, D_{i} D_{\bar{j}} Z_{r}\right)-Z_{\bar{j}} Z_{i}\left(g_{r \bar{r}}\right)+Z_{\bar{j}}\left(g\left(D_{i} Z_{r}, Z_{\bar{r}}\right)\right) \\
& =-g\left(D_{i} Z_{\bar{r}}, D_{\bar{j}} Z_{r}\right)+g\left(\left[Z_{\bar{j}}, Z_{i}\right] Z_{r}, Z_{\bar{r}}\right)+g\left(D_{i} Z_{r}, D_{\bar{j}} Z_{\bar{r}}\right)-Z_{\bar{j}} Z_{i}\left(g_{\bar{r}}\right), \\
Z_{i}( & \left.g\left(Z_{\bar{j}}, D_{r} Z_{\bar{r}}\right)\right)+Z_{\bar{j}}\left(g\left(Z_{i}, D_{r} Z_{\bar{r}}\right)\right) \\
= & g\left(D_{i} Z_{\bar{j}}, D_{r} Z_{\bar{r}}\right)+g\left(Z_{\bar{j}}, D_{i} D_{r} Z_{\bar{r}}\right)+g\left(D_{\bar{j}} Z_{i}, D_{r} Z_{\bar{r}}\right)+g\left(Z_{i}, D_{\bar{j}} D_{r} Z_{\bar{r}}\right) \\
= & g\left(D_{i} Z_{\bar{j}}, D_{r} Z_{\bar{r}}\right)+g\left(D_{\bar{j}} Z_{i}, D_{r} Z_{\bar{r}}\right)+g\left(Z_{\bar{j}},\left[Z_{i}, Z_{r}\right] Z_{\bar{r}}\right)+g\left(Z_{\bar{j}}, D_{r} D_{i} Z_{\bar{r}}\right) \\
& +g\left(Z_{i},\left[Z_{\bar{j}}, Z_{r}\right] Z_{\bar{r}}\right)+g\left(Z_{i}, D_{r} D_{\bar{j}} Z_{\bar{r}}\right) \\
= & g\left(D_{i} Z_{\bar{j}}, D_{r} Z_{\bar{r}}\right)+g\left(D_{\bar{j}} Z_{i}, D_{r} Z_{\bar{r}}\right)+g\left(Z_{\bar{j}},\left[Z_{i}, Z_{r}\right] Z_{\bar{r}}\right)+g\left(Z_{i},\left[Z_{\bar{j}}, Z_{r}\right] Z_{\bar{r}}\right) \\
& +g\left(Z_{\bar{j}}, D_{r}\left[Z_{i}, Z_{\bar{r}}\right]\right)+g\left(Z_{\bar{j}}, D_{r} D_{\bar{r}} Z_{i}\right)+g\left(Z_{i}, D_{r}\left[Z_{\bar{j}}, Z_{\bar{r}}\right]\right)+g\left(Z_{i}, D_{r} D_{\bar{r}} Z_{\bar{j}}\right) \\
= & g\left(D_{i} Z_{\bar{j}}, D_{r} Z_{\bar{r}}\right)+g\left(D_{\bar{j}} Z_{i}, D_{r} Z_{\bar{r}}\right)+g\left(Z_{\bar{j}},\left[Z_{i}, Z_{r}\right] Z_{\bar{r}}\right)+g\left(Z_{i},\left[Z_{\bar{j}}, Z_{r}\right] Z_{\bar{r}}\right) \\
& +g\left(Z_{\bar{j}}, D_{r}\left[Z_{i}, Z_{\bar{r}}\right]\right)+g\left(Z_{i}, D_{r}\left[Z_{\bar{j}}, Z_{\bar{r}}\right]\right) \\
& -g\left(D_{\bar{r}} Z_{i}, D_{r} Z_{\bar{j}}\right)-g\left(D_{r} Z_{i}, D_{\bar{r}} Z_{\bar{j}}\right)+Z_{r} Z_{\bar{r}}\left(g_{i \bar{j}}\right)
\end{aligned}
$$




$$
\begin{aligned}
= & g\left(D_{i} Z_{\bar{j}}, D_{r} Z_{\bar{r}}\right)+g\left(D_{\bar{j}} Z_{i}, D_{r} Z_{\bar{r}}\right)+g\left(Z_{\bar{j}},\left[Z_{i}, Z_{r}\right] Z_{\bar{r}}\right)+g\left(Z_{i},\left[Z_{\bar{j}}, Z_{r}\right] Z_{\bar{r}}\right) \\
& +Z_{r}\left(g\left(Z_{\bar{j}},\left[Z_{i}, Z_{\bar{r}}\right]\right)\right)-g\left(D_{r} Z_{\bar{j}},\left[Z_{i}, Z_{\bar{r}}\right]\right)+Z_{r}\left(g\left(Z_{i},\left[Z_{\bar{j}}, Z_{\bar{r}}\right]\right)\right) \\
& -g\left(D_{r} Z_{i},\left[Z_{\bar{j}}, Z_{\bar{r}}\right]\right)-g\left(D_{\bar{r}} Z_{i}, D_{r} Z_{\bar{j}}\right)-g\left(D_{r} Z_{i}, D_{\bar{r}} Z_{\bar{j}}\right)+Z_{r} Z_{\bar{r}}\left(g_{i \bar{j}}\right),
\end{aligned}
$$

where we used that

$$
\begin{aligned}
g\left(Z_{i}, D_{r} D_{\bar{r}} Z_{\bar{j}}\right) & =Z_{r}\left(g\left(Z_{i}, D_{\bar{r}} Z_{\bar{j}}\right)\right)-g\left(D_{r} Z_{i}, D_{\bar{r}} Z_{\bar{j}}\right) \\
& =Z_{r} Z_{\bar{r}}\left(g_{i \bar{j}}\right)-Z_{r}\left(g\left(D_{\bar{r}} Z_{i}, Z_{\bar{j}}\right)\right)-g\left(D_{r} Z_{i}, D_{\bar{r}} Z_{\bar{j}}\right) \\
& =Z_{r} Z_{\bar{r}}\left(g_{i \bar{j}}\right)-g\left(D_{r} D_{\bar{r}} Z_{i}, Z_{\bar{j}}\right)-g\left(D_{\bar{r}} Z_{i}, D_{r} Z_{\bar{j}}\right)-g\left(D_{r} Z_{i}, D_{\bar{r}} Z_{\bar{j}}\right)
\end{aligned}
$$

and

$$
\begin{aligned}
& g\left(Z_{\bar{j}}, D_{r}\left[Z_{i}, Z_{\bar{r}}\right]\right)=Z_{r}\left(g\left(Z_{\bar{j}},\left[Z_{i}, Z_{\bar{r}}\right]\right)\right)-g\left(D_{r} Z_{\bar{j}},\left[Z_{i}, Z_{\bar{r}}\right]\right), \\
& g\left(Z_{i}, D_{r}\left[Z_{\bar{j}}, Z_{\bar{r}}\right]\right)=Z_{r}\left(g\left(Z_{i},\left[Z_{\bar{j}}, Z_{\bar{r}}\right]\right)\right)-g\left(D_{r} Z_{i},\left[Z_{\bar{j}}, Z_{\bar{r}}\right]\right) .
\end{aligned}
$$

We also compute

$$
\begin{aligned}
Z_{r} & \left(g\left(Z_{i},\left[Z_{\bar{j}}, Z_{\bar{r}}\right]\right)\right)=g\left(D_{r} Z_{i},\left[Z_{\bar{j}}, Z_{\bar{r}}\right]\right)+g\left(Z_{i}, D_{r}\left[Z_{\bar{j}}, Z_{\bar{r}}\right]\right), \\
D_{r}\left[Z_{\bar{j}}, Z_{\bar{r}}\right] & =D_{r}\left(D_{\bar{j}} Z_{\bar{r}}-D_{\bar{r}} Z_{\bar{j}}\right) \\
& =\left[Z_{r}, Z_{\bar{j}}\right] Z_{\bar{r}}+D_{\bar{j}}\left[Z_{r}, Z_{\bar{r}}\right]-\left[Z_{r}, Z_{\bar{r}}\right] Z_{\bar{j}}-D_{\bar{r}}\left[Z_{r}, Z_{\bar{j}}\right]+\left[Z_{\bar{j}}, Z_{\bar{r}}\right] Z_{r}
\end{aligned}
$$

and

$$
\begin{aligned}
Z_{r}( & \left.g\left(Z_{i},\left[Z_{\bar{j}}, Z_{\bar{r}}\right]\right)\right) \\
= & g\left(D_{r} Z_{i},\left[Z_{\bar{j}}, Z_{\bar{r}}\right]\right)+g\left(Z_{i},\left[Z_{r}, Z_{\bar{j}}\right] Z_{\bar{r}}\right)+g\left(Z_{i}, D_{\bar{j}}\left[Z_{r}, Z_{\bar{r}}\right]\right) \\
& -g\left(Z_{i},\left[Z_{r}, Z_{\bar{r}}\right] Z_{\bar{j}}\right)-g\left(Z_{i}, D_{\bar{r}}\left[Z_{r}, Z_{\bar{j}}\right]\right)+g\left(Z_{i},\left[Z_{\bar{j}}, Z_{\bar{r}}\right] Z_{r}\right) \\
= & g\left(D_{r} Z_{i},\left[Z_{\bar{j}}, Z_{\bar{r}}\right]\right)+g\left(Z_{i},\left[Z_{r}, Z_{\bar{j}}\right] Z_{\bar{r}}\right)+Z_{\bar{j}}\left(g\left(Z_{i},\left[Z_{r}, Z_{\bar{r}}\right]\right)\right) \\
& -g\left(D_{\bar{j}} Z_{i},\left[Z_{r}, Z_{\bar{r}}\right]\right)-g\left(Z_{i},\left[Z_{r}, Z_{\bar{r}}\right] Z_{\bar{j}}\right)-Z_{\bar{r}}\left(g\left(Z_{i},\left[Z_{r}, Z_{\bar{j}}\right]\right)\right) \\
& +g\left(D_{\bar{r}} Z_{i},\left[Z_{r}, Z_{\bar{j}}\right]\right)+g\left(Z_{i},\left[Z_{\bar{j}}, Z_{\bar{r}}\right] Z_{r}\right) \\
= & g\left(D_{r} Z_{i},\left[Z_{\bar{j}}, Z_{\bar{r}}\right]\right)+g\left(Z_{i},\left[Z_{r}, Z_{\bar{j}}\right] Z_{\bar{r}}\right)-g\left(D_{\bar{j}} Z_{i},\left[Z_{r}, Z_{\bar{r}}\right]\right) \\
& -g\left(Z_{i},\left[Z_{r}, Z_{\bar{r}}\right] Z_{\bar{j}}\right)+g\left(D_{\bar{r}} Z_{i},\left[Z_{r}, Z_{\bar{j}}\right]\right)+g\left(Z_{i},\left[Z_{\bar{j}}, Z_{\bar{r}}\right] Z_{r}\right) \\
& +Z_{\bar{j}}\left(B_{r \bar{r}}^{\bar{s}}\right) g_{i \bar{s}}-Z_{\bar{r}}\left(B_{r \bar{j}}^{\bar{s}}\right) g_{i \bar{s}}+\mathcal{O}(\bar{Z}(g)),
\end{aligned}
$$

where $\mathcal{O}(\bar{Z}(g))$ denotes the set of all terms including $\bar{Z}(g)$ and we used that

$$
\begin{aligned}
Z_{\bar{j}} & \left(g\left(Z_{i},\left[Z_{r}, Z_{\bar{r}}\right]\right)\right)-Z_{\bar{r}}\left(g\left(Z_{i},\left[Z_{r}, Z_{\bar{j}}\right]\right)\right) \\
\quad & =Z_{\bar{j}}\left(g\left(Z_{i}, B_{r \bar{r}}^{s} Z_{s}+B_{r \bar{r}}^{\bar{s}} Z_{\bar{s}}\right)\right)-Z_{\bar{r}}\left(g\left(Z_{i}, B_{r \bar{j}}^{s} Z_{s}+B_{r \bar{j}}^{\bar{s}} Z_{\bar{s}}\right)\right) \\
& =Z_{\bar{j}}\left(B_{r \bar{r}}^{\bar{s}} g_{i \bar{s}}\right)-Z_{\bar{r}}\left(B_{r \bar{j}}^{\bar{s}} g_{i \bar{s}}\right) \\
& =Z_{\bar{j}}\left(B_{r \bar{r}}^{\bar{s}}\right) g_{i \bar{s}}-Z_{\bar{r}}\left(B_{r \bar{j}}^{\bar{s}}\right) g_{i \bar{s}}+\mathcal{O}(\bar{Z}(g)) .
\end{aligned}
$$


Here note that we actually have $g\left(Z_{i},\left[Z_{r}, Z_{\bar{r}}\right]\right)=\mathcal{O}(g, \partial J)$ since $Z_{r}$ is constructed by projecting local coordinates onto $T^{1,0} M$ using $J$ (cf. [7, Proposition 5.5]).

Then we compute using the formulae above,

$$
\begin{aligned}
& \left(\partial \partial_{g}^{*} \omega\right)_{i} \bar{j}+\left(\bar{\partial} \bar{\partial}_{g}^{*} \omega\right)_{i} \bar{j} \\
& =\sqrt{-1}\left\{-Z_{i}\left(w_{\bar{j}}\right)-Z_{\bar{j}}\left(w_{i}\right)+B_{i \bar{j}}^{\bar{r}} w_{\bar{r}}+B_{\bar{j} i}^{r} w_{r}\right\} \\
& =\sqrt{-1}\left\{Z_{r} Z_{\bar{r}}\left(g_{i \bar{j}}\right)-Z_{\bar{j}} Z_{i}\left(g_{r \bar{r}}\right)-g\left(D_{i} Z_{\bar{r}}, D_{\bar{j}} Z_{r}\right)+g\left(\left[Z_{\bar{j}}, Z_{i}\right] Z_{r}, Z_{\bar{r}}\right)+g\left(D_{i} Z_{r}, D_{\bar{j}} Z_{\bar{r}}\right)\right. \\
& +g\left(D_{i} Z_{\bar{j}}, D_{r} Z_{\bar{r}}\right)+g\left(D_{\bar{j}} Z_{i}, D_{r} Z_{\bar{r}}\right)+g\left(Z_{\bar{j}},\left[Z_{i}, Z_{r}\right] Z_{\bar{r}}\right)+g\left(Z_{i},\left[Z_{\bar{j}}, Z_{r}\right] Z_{\bar{r}}\right) \\
& +Z_{r}\left(g\left(Z_{\bar{j}},\left[Z_{i}, Z_{\bar{r}}\right]\right)\right)-g\left(D_{r} Z_{\bar{j}},\left[Z_{i}, Z_{\bar{r}}\right]\right)+Z_{r}\left(g\left(Z_{i},\left[Z_{\bar{j}}, Z_{\bar{r}}\right]\right)\right)-g\left(D_{r} Z_{i},\left[Z_{\bar{j}}, Z_{\bar{r}}\right]\right) \\
& -g\left(D_{\bar{r}} Z_{i}, D_{r} Z_{\bar{j}}\right)-g\left(D_{r} Z_{i}, D_{\bar{r}} Z_{\bar{j}}\right)+Z_{i}\left(g\left(Z_{\bar{j}},\left[Z_{\bar{r}}, Z_{r}\right]\right)\right) \\
& -Z_{i}\left(B_{\bar{r} r}^{k}\right) g_{k \bar{j}}+Z_{i}\left(B_{\bar{j} r}^{k}\right) g_{k \bar{r}}-Z_{\bar{j}}\left(B_{r \bar{r}}^{\bar{k}}\right) g_{i \bar{k}}+Z_{\bar{j}}\left(B_{i \bar{r}}^{\bar{k}}\right) g_{r \bar{k}} \\
& \left.-B_{\bar{r} r}^{k} Z_{i}\left(g_{k \bar{j}}\right)+B_{\bar{j} r}^{k} Z_{i}\left(g_{k \bar{r}}\right)-B_{r \bar{r}}^{\bar{k}} Z_{\bar{j}}\left(g_{i \bar{k}}\right)+B_{i \bar{r}}^{\bar{k}} Z_{\bar{j}}\left(g_{r \bar{k}}\right)+B_{i \bar{j}}^{\bar{r}} w_{\bar{r}}+B_{\bar{j} i}^{r} w_{r}\right\} \\
& =\sqrt{-1}\left\{Z_{r} Z_{\bar{r}}\left(g_{i \bar{j}}\right)-Z_{\bar{j}} Z_{i}\left(g_{r \bar{r}}\right)-g\left(D_{i} Z_{\bar{r}}, D_{\bar{j}} Z_{r}\right)+g\left(\left[Z_{\bar{j}}, Z_{i}\right] Z_{r}, Z_{\bar{r}}\right)+g\left(D_{i} Z_{r}, D_{\bar{j}} Z_{\bar{r}}\right)\right. \\
& +g\left(D_{i} Z_{\bar{j}}, D_{r} Z_{\bar{r}}\right)+g\left(D_{\bar{j}} Z_{i}, D_{r} Z_{\bar{r}}\right)+g\left(Z_{\bar{j}},\left[Z_{i}, Z_{r}\right] Z_{\bar{r}}\right)+g\left(Z_{i},\left[Z_{\bar{j}}, Z_{r}\right] Z_{\bar{r}}\right) \\
& -g\left(D_{r} Z_{\bar{j}},\left[Z_{i}, Z_{\bar{r}}\right]\right)+g\left(D_{r} Z_{i},\left[Z_{\bar{j}}, Z_{\bar{r}}\right]\right)+g\left(Z_{i},\left[Z_{r}, Z_{\bar{j}}\right] Z_{\bar{r}}\right)-g\left(D_{\bar{j}} Z_{i},\left[Z_{r}, Z_{\bar{r}}\right]\right) \\
& -g\left(Z_{i},\left[Z_{r}, Z_{\bar{r}}\right] Z_{\bar{j}}\right)+g\left(D_{\bar{r}} Z_{i},\left[Z_{r}, Z_{\bar{j}}\right]\right)+g\left(Z_{i},\left[Z_{\bar{j}}, Z_{\bar{r}}\right] Z_{r}\right)-g\left(D_{r} Z_{i},\left[Z_{\bar{j}}, Z_{\bar{r}}\right]\right) \\
& -g\left(D_{\bar{r}} Z_{i}, D_{r} Z_{\bar{j}}\right)-g\left(D_{r} Z_{i}, D_{\bar{r}} Z_{\bar{j}}\right)+Z_{\bar{j}}\left(B_{i \bar{r}}^{\bar{k}}\right) g_{r \bar{k}}+Z_{i}\left(B_{\bar{j} r}^{k}\right) g_{k \bar{r}}+B_{i \bar{j}}^{\bar{r}} w_{\bar{r}}+B_{\bar{j} i}^{r} w_{r} \\
& \left.-Z_{\bar{r}}\left(B_{r \bar{j}}^{\bar{s}}\right) g_{i \bar{s}}+Z_{r}\left(B_{i \bar{r}}^{S}\right) g_{s \bar{j}}+\mathcal{O}(Z(g))+\mathcal{O}(\bar{Z}(g))\right\},
\end{aligned}
$$

where $\mathcal{O}(Z(g))$ is the set of all terms including $Z(g)$ and we used $(\dagger)$ at the third equality and also that

$$
Z_{r}\left(g\left(Z_{\bar{j}},\left[Z_{i}, Z_{\bar{r}}\right]\right)\right)+Z_{i}\left(g\left(Z_{\bar{j}},\left[Z_{\bar{r}}, Z_{r}\right]\right)\right)=Z_{r}\left(B_{i \bar{r}}^{S}\right) g_{s \bar{j}}+Z_{i}\left(B_{\bar{r} r}^{S}\right) g_{s \bar{j}}+\mathcal{O}(Z(g)) .
$$

Therefore, $\partial \partial_{g}^{*} \omega+\bar{\partial} \bar{\partial}_{g}^{*} \omega$ involves the second derivatives of $g ; Z_{r} Z_{\bar{r}}\left(g_{i \bar{j}}\right)-Z_{\bar{j}} Z_{i}\left(g_{r \bar{r}}\right)$.

We also have the following.

\section{LEMMA 3.4. One has}

$$
\begin{aligned}
P_{i \bar{j}}= & -g^{k \bar{l}} Z_{\bar{j}} Z_{i}\left(g_{k \bar{l}}\right)+Z_{i}\left(B_{\bar{j} r}^{r}\right)+Z_{\bar{j}}\left(B_{i \bar{r}}^{\bar{r}}\right)+B^{\circ} * B^{\circ}+\Gamma * \bar{\Gamma}+B^{\circ} * \Gamma \\
& +\mathcal{O}(Z(g))+\mathcal{O}(\bar{Z}(g)) .
\end{aligned}
$$

Proof. We compute using the formula in Lemma 2.3,

$$
\begin{aligned}
P_{i \bar{j}}= & g^{k \bar{l}} R_{i \bar{j} k \bar{l}} \\
= & g^{k \bar{l}} g\left(\nabla_{i} \nabla_{\bar{j}} Z_{k}-\nabla_{\bar{j}} \nabla_{i} Z_{k}-\nabla_{\left[Z_{i}, Z_{\bar{j}}\right]} Z_{k}, Z_{\bar{l}}\right) \\
= & g^{k \bar{l}}\left(Z_{i}\left(g\left(\nabla_{\bar{j}} Z_{k}, Z_{\bar{l}}\right)\right)-g\left(\nabla_{\bar{j}} Z_{k}, \nabla_{i} Z_{\bar{l}}\right)-Z_{\bar{j}}\left(g\left(\nabla_{i} Z_{k}, Z_{\bar{l}}\right)\right)\right. \\
& \left.+g\left(\nabla_{i} Z_{k}, \nabla_{\bar{j}} Z_{\bar{l}}\right)-B_{i \bar{j}}^{r} g\left(\nabla_{r} Z_{k}, Z_{\bar{l}}\right)-B_{i \bar{j}}^{\bar{r}_{-}} g\left(\nabla_{\bar{r}} Z_{k}, Z_{\bar{l}}\right)\right)
\end{aligned}
$$




$$
\begin{aligned}
= & g^{k \bar{l}}\left(Z_{i}\left(g\left(\nabla_{\bar{j}} Z_{k}, Z_{\bar{l}}\right)\right)-B_{\bar{j} k}^{r} B_{i \bar{l}}^{\bar{s}} g_{r \bar{s}}-Z_{\bar{j}} Z_{i}\left(g\left(Z_{k}, Z_{\bar{l}}\right)\right)+Z_{\bar{j}}\left(g\left(Z_{k}, \nabla_{i} Z_{\bar{l}}\right)\right)\right. \\
& \left.+\Gamma_{i k}^{r} \Gamma_{\bar{j} \bar{l}}^{\bar{s}} g_{r \bar{s}}-B_{i \bar{j}}^{r} \Gamma_{r k}^{s} g_{s \bar{l}}-B_{i \bar{j}}^{\bar{r}} B_{\bar{r} k}^{s} g_{s \bar{l}}\right) \\
= & -g^{k \bar{l}} Z_{\bar{j}} Z_{i}\left(g_{k \bar{l}}\right)+Z_{i}\left(B_{\bar{j} r}^{r}\right)+Z_{\bar{j}}\left(B_{i \bar{r}}^{\bar{r}}\right)+B^{\circ} * B^{\circ}+\Gamma * \bar{\Gamma}+B^{\circ} * \Gamma \\
& +\mathcal{O}(Z(g))+\mathcal{O}(\bar{Z}(g)) .
\end{aligned}
$$

Hence, from Lemma 3.4, $\Phi(\omega)_{i} \bar{j}=-\left(\partial \partial_{g}^{*} \omega\right)_{i} \bar{j}-\left(\bar{\partial} \bar{\partial}_{g}^{*} \omega\right)_{i} \bar{j}+\sqrt{-1} P_{i \bar{j}}$ involves only one term of the second derivatives of $g,-Z_{r} Z_{\bar{r}}\left(g_{i} \bar{j}\right)$,

$$
\begin{aligned}
-g^{k \bar{l}} Z_{k} Z_{\bar{l}}\left(g_{i} \bar{j}\right) & =-g^{k \bar{l}}\left(Z_{k} Z_{\bar{l}}-\left[Z_{k}, Z_{\bar{l}}\right]^{(0,1)}\right) g_{i \bar{j}}-g^{k \bar{l}}\left[Z_{k}, Z_{\bar{l}}\right]^{(0,1)}\left(g_{i \bar{j}}\right) \\
& =-g^{k \bar{l}} \partial_{k} \partial_{\bar{l}} g_{i \bar{j}}+\mathcal{O}(\bar{Z}(g)),
\end{aligned}
$$

which implies that the operator $\Phi$ is strictly elliptic since $g$ is positive definite.

\section{The generalized identifiability theorem}

In order to prove Theorem 1.2, we prepare the following lemma.

LEMMA 4.1. The following formula holds for any almost Hermitian metric:

$$
\left(\operatorname{div}^{\nabla} T^{\prime}\right)_{i \bar{j}}=-(\bar{\nabla} w)_{i \bar{j}}-B T_{i \bar{j}}^{\prime}-\bar{Z}\left(T^{\prime}\right)_{i \bar{j}}
$$

Proof. Let $\left\{Z_{r}\right\}$ be a local unitary $(1,0)$-frame with respect to $g$. Using the equality $Z_{\bar{q}}\left(g_{r} \bar{j}\right)=0=Z_{\bar{j}}\left(g_{r \bar{q}}\right)$ with respect to such a frame in the fourth line below, we compute

$$
\begin{aligned}
\left(\operatorname{div}^{\nabla} T^{\prime}\right)_{i \bar{j}}= & g^{p \bar{q}} \nabla_{\bar{q}} T_{p i \bar{j}} \\
= & g^{p \bar{q}}\left(Z_{\bar{q}}\left(T_{p i}\right)-\Gamma_{\bar{q} p}^{r} T_{r i \bar{j}}-\Gamma_{\bar{q} i}^{r} T_{p r \bar{j}}-\Gamma_{\bar{q} \bar{j}}^{\bar{r}} T_{p i \bar{r}}\right) \\
= & g^{p \bar{q}}\left(Z_{\bar{q}}\left(T_{p i}^{r}\right) g_{r \bar{j}}+T_{p i}^{r} Z_{\bar{q}}\left(g_{r \bar{j}}\right)-\Gamma_{\bar{q} p}^{r} T_{r i \bar{j}}-\Gamma_{\bar{q} i}^{r} T_{p r \bar{j}}-\Gamma_{\bar{q} \bar{j}}^{\bar{r}} T_{p i \bar{r}}\right) \\
= & g^{p \bar{q}}\left(Z_{\bar{q}}\left(T_{p i}^{r}\right) g_{r \bar{j}}+T_{p i}^{r} Z_{\bar{j}}\left(g_{r \bar{q}}\right)-\Gamma_{\bar{q} p}^{r} T_{r i \bar{j}}-\Gamma_{\bar{q} i}^{r} T_{p r \bar{j}}-\Gamma_{\bar{q} \bar{j}}^{\bar{r}} T_{p i \bar{r}}\right) \\
= & g^{p \bar{q}}\left(Z_{\bar{q}}\left(T_{p i}^{r}\right) g_{r \bar{j}}+Z_{\bar{j}}\left(T_{p i}^{r} g_{r \bar{q}}\right)-Z_{\bar{j}}\left(T_{p i}^{r}\right) g_{r \bar{q}}-\Gamma_{\bar{q} p}^{r} T_{r i \bar{j}}-\Gamma_{\bar{q} i}^{r} T_{p r} \bar{j}-\Gamma_{\bar{q} \bar{j}}^{\bar{r}} T_{p i \bar{r}}\right) \\
= & g^{p \bar{q}}\left(Z_{\bar{q}}\left(T_{p i}^{r}\right) g_{r \bar{j}}-\nabla_{\bar{j}} T_{i p \bar{q}}+\Gamma_{\bar{j} p}^{r} T_{r i \bar{q}}+\Gamma_{\bar{j} i}^{r} T_{p r \bar{q}}+\Gamma_{\bar{j} \bar{q}}^{\bar{r}} T_{p i \bar{r}}-Z_{\bar{j}}\left(T_{p i}^{r}\right) g_{r \bar{q}}\right. \\
& \left.-\Gamma_{\bar{q} p}^{r} T_{r i \bar{j}}-\Gamma_{\bar{q} i}^{r} T_{p r} \bar{j}-\Gamma_{\bar{q} \bar{j}}^{\bar{r}} T_{p i \bar{r}}\right) \\
= & g^{p \bar{q}}\left(Z_{\bar{q}}\left(T_{p i}^{r}\right) g_{r \bar{j}}-\nabla_{\bar{j}} T_{i p \bar{q}}+\Gamma_{\bar{j} i}^{r} T_{p r \bar{q}}-Z_{\bar{j}}\left(T_{p i}^{r} g_{r \bar{q}}\right)+T_{p i}^{r} Z_{\bar{j}}\left(g_{r \bar{q}}\right)\right. \\
& \left.-\Gamma_{\bar{q} p}^{r} T_{r i \bar{j}}-\Gamma_{\bar{q} i}^{r} T_{p r} \bar{j}+\left(\Gamma_{\bar{j} \bar{q}}^{\bar{r}}-\Gamma_{\bar{q} \bar{j}}^{\bar{r}}-B_{\bar{j} \bar{q}}^{\bar{r}}\right) T_{p i \bar{r}}+\Gamma_{\bar{j} p}^{r} T_{r i \bar{q}}+B_{\bar{j} \bar{q}}^{\bar{r}} T_{p i \bar{r}}\right) \\
= & -\nabla_{\bar{j}} w_{i}-B_{\bar{r} p}^{j} T_{i r \bar{p}}-B_{\bar{p} i}^{r} T_{p r} \bar{j}-B_{\bar{r} r}^{p} T_{p i \bar{j}}-B_{\bar{j} i}^{r} w_{r} \\
& +Z_{\bar{r}}\left(T_{r i}^{s}\right) g_{s} \bar{j}+Z_{\bar{j}}\left(w_{i}\right)+g^{p \bar{q}} T_{p i}^{r} Z_{\bar{j}}\left(g_{r \bar{q}}\right) \\
= & \left.-(\bar{\nabla} w)_{i \bar{j}}-B T_{i \bar{j}}^{\prime}-\bar{Z}_{\left(T^{\prime}\right.}\right)_{i \bar{j}},
\end{aligned}
$$


where we used the following in the eighth line:

$$
\begin{aligned}
g^{p \bar{q}} & \left(\left(\Gamma_{\bar{j} \bar{q}}^{\bar{r}}-\Gamma_{\bar{q} \bar{j}}^{\bar{r}}-B_{\bar{j} \bar{q}}^{\bar{r}}\right) T_{p i \bar{r}}+\Gamma_{\bar{j} p}^{r} T_{r i \bar{q}}+B_{\bar{j} \bar{q}}^{\bar{r}} T_{p i \bar{r}}\right) \\
& =T_{\bar{j} \bar{p}}^{\bar{r}} T_{p i \bar{r}}-\left(B_{\bar{j} p}^{r}+B_{\bar{j} \bar{r}}^{\bar{p}}\right) T_{i r \bar{p}} \\
& \left.=-T_{\bar{j} \bar{p} r} T_{i p \bar{r}}-\overline{\left(B_{j \bar{p}}^{\bar{r}}+B_{j r}^{p}-B_{r \bar{p}}^{\bar{j}}\right.}\right) T_{i r \bar{p}}-B_{\bar{r} p}^{j} T_{i r \bar{p}} \\
& =-Q_{i \bar{j}}^{1}+\overline{\left(\Gamma_{j r}^{p}-\Gamma_{r j}^{p}-B_{j r}^{p}\right)} T_{i r \bar{p}}-B_{\bar{r} p}^{j} T_{i r \bar{p}} \\
& =-Q_{i \bar{j}}^{1}+Q_{i \bar{j}}^{1}-B_{\bar{r} p}^{j} T_{i r \bar{p}} \\
& =-B_{\bar{r} p}^{j} T_{i r \bar{p}} .
\end{aligned}
$$

Here we used $B_{j \bar{p}}^{\bar{r}}=\Gamma_{j \bar{p}}^{\bar{r}}=-\Gamma_{j r}^{p}$ with respect to a unitary frame in the third line above.

Combining the formula above with the formula in Lemma 2.5 , we obtain the following desired result.

Proposition 4.1. Let $(M, \omega(t), J)$ be a solution to $(A H F)$ starting at the initial almost Hermitian metric $\omega_{0}$. Then we have

$$
\frac{\partial}{\partial t} \omega(t)=-S(\omega(t))-Q^{7}(\omega(t))-Q^{8}(\omega(t))+B T^{\prime}(\omega(t))+\bar{Z}\left(T^{\prime}\right)(\omega(t)) .
$$

Proof. Taking into account Lemma 3.3, the flow (AHF) takes the expression

$$
\begin{aligned}
\frac{\partial}{\partial t} \omega & =\partial \partial_{g}^{*} \omega+\bar{\partial} \bar{\partial}_{g}^{*} \omega-P \\
& =-\nabla \bar{w}-\bar{\nabla} w-P .
\end{aligned}
$$

From the result of Lemma 2.3, we have

$$
P=\operatorname{div}^{\nabla} T^{\prime}-\nabla \bar{w}+S+Q^{7}+Q^{8} .
$$

Applying the result of Lemma 4.1 yields

$$
P=-\bar{\nabla} w-B T^{\prime}-\bar{Z}\left(T^{\prime}\right)-\nabla \bar{w}+S+Q^{7}+Q^{8} .
$$

Hence, we conclude that

$$
\frac{\partial}{\partial t} \omega=-S-Q^{7}-Q^{8}+B T^{\prime}+\bar{Z}\left(T^{\prime}\right)
$$

Hence we have proved the second main result.

\section{REFERENCES}

[1] X.-Q. Fan, L.-F. Tam and C.-J. Yu. Product of almost Hermitian manifolds. J. Geom. Anal. 24 (2014), 1425-1446.

[2] P. Gauduchon. Hermitian connections and Dirac operators. Boll. Unione. Mat. Ital. B (7) (suppl.) 11(2) (1997), 257-288.

[3] J. Streets. Geometric flows in complex geometry. Lecture notes at the Hefei Advanced School on PDEs in Geometry and Physics, 30 June-11 July 2014. 
[4] J. Streets and G. Tian. A parabolic flow of pluriclosed metrics. Int. Math. Res. Not. IMRN 2010(16) (2010), 3101-3133.

[5] J. Streets and G. Tian. Hermitian curvature flow. J. Eur. Math. Soc. (JEMS) 13(3) (2011), 601-634.

[6] J. Streets and G. Tian. Regularity results for pluriclosed flow. C. R. Math. Acad. Sci. Paris 349(1-2) (2011), 14.

[7] J. Streets and G. Tian. Symplectic curvature flow. J. reine angew. Math. 2014(696) (2014), 143-185.

[8] L. Vezzoni. On Hermitian curvature flow on almost complex manifolds. Differential. Geom. Appl. 29 (2011), 709-722.

[9] B. Yang and F. Zheng. On curvature tensors of Hermitian manifolds. Preprint, 2016, arXiv:1602.01189v1.

[10] C.-J. Yu. Curvature identities on almost Hermitian manifolds and applications. Sci. China Math. 60(2) (2017), 285-300.

[11] T. Zheng. An almost complex Chern-Ricci flow. J. Geom. Anal. 28 (2017), 2129-2165.

\author{
Masaya Kawamura \\ Department of Social Design Engineering \\ National Institute of Technology, Kochi College \\ 200-1, Otsu, Monobe, Nankoku \\ Kochi 783-8508 \\ Japan \\ (E-mail: kawamura@kochi-ct.ac.jp)
}

